ARTICLE

https://doi.org/10.1038/s41467-019-11879-2

\title{
Dynamic changes in epithelial cell morphology control thymic organ size during atrophy and regeneration
}

\author{
Thomas Venables ${ }^{1,3}$, Ann V. Griffith (10 1,2,3, Alice DeAraujo ${ }^{1} \&$ Howard T. Petrie $^{1}$
}

T lymphocytes must be produced throughout life, yet the thymus, where $T$ lymphocytes are made, exhibits accelerated atrophy with age. Even in advanced atrophy, however, the thymus remains plastic, and can be regenerated by appropriate stimuli. Logically, thymic atrophy is thought to reflect senescent cell death, while regeneration requires proliferation of stem or progenitor cells, although evidence is scarce. Here we use conditional reporters to show that accelerated thymic atrophy reflects contraction of complex cell projections unique to cortical epithelial cells, while regeneration requires their regrowth. Both atrophy and regeneration are independent of changes in epithelial cell number, suggesting that the size of the thymus is regulated primarily by rate-limiting morphological changes in cortical stroma, rather than by their cell death or proliferation. Our data also suggest that cortical epithelial morphology is under the control of medullary stromal signals, revealing a previously unrecognized endocrine-paracrine signaling axis in the thymus.

\footnotetext{
${ }^{1}$ The Scripps Research Institute, 130 Scripps Way, Jupiter, FL 33458, USA. ${ }^{2}$ Present address: Department of Microbiology, Immunology and Molecular Genetics, UT Health San Antonio, San Antonio, TX 78229, USA. ${ }^{3}$ These authors contributed equally: Thomas Venables, Ann V. Griffith. Correspondence and requests for materials should be addressed to H.T.P. (email: htpetrie@gmail.com)
} 
ike all blood cells, T lymphocytes are lost throughout life, and must be continuously replaced. The thymus is the primary site of post-natal T lymphopoiesis. However, unlike other tissues that undergo steady-state differentiation, there are no lymphoid stem cells contained within the thymus, and instead it depends on recruitment of multipotent progenitors from the blood $^{1}$. Upon entering the thymus, microenvironmental conditions unique to the organ specify $\mathrm{T}$ lineage commitment, as well as proliferation, tolerance, $\mathrm{T}$ lineage divergence, and other processes. Ultimately, new T lymphocytes are exported to the periphery. Since the hematolymphoid components of the thymus are quite transient, it is obvious that they cannot define its durable identity. Instead, this identity is established by the stromal components of the thymus, primarily including specialized thymic epithelial cells (TEC, reviewed in refs. ${ }^{2,3}$ ). TEC provide most of the signals that induce and control $\mathrm{T}$ lymphocyte production (reviewed in ref. ${ }^{4}$ ). Reciprocally, they depend on the presence of lymphocytes for their differentiation and organization ${ }^{5-7}$, a process referred to as crosstalk. Cortical TEC (cTEC) also establish the physical matrix for directional migration of lymphoid progenitor cells within the thymus ${ }^{8,9}$, simultaneously limiting thymus size by providing a rate limiting number of progenitor niches in this matrix ${ }^{10}$. Medullary TEC (mTEC) are essential in imposing self-tolerance in new $\mathrm{T}$ cells prior to export to the periphery ${ }^{11}$, and are likely to play roles in $\mathrm{T}$ lineage divergence and functional maturation as well. Thus, epithelial cells exhibit diverse essential roles in maintaining $\mathrm{T}$ cell production and function.

In other tissues, epithelial cells exhibit distinctive squamous, cuboidal, or columnar morphologies, and are polarized (with respect to a basement membrane) in either a single simple layer or in multiple stratified layers ${ }^{12}$. In the thymus, with the exception of a small proportion of conventional epithelial cells lining the capsule and blood vessels, most TEC lack classical epithelial morphology ${ }^{13-16}$. Instead, they are defined as epithelial mainly based on biochemical features, such as the appearance of desmosomes or keratin filaments ${ }^{13,15,17}$. Various histochemical markers indicate that CTEC, in particular, form an extensive network of finely branched cell processes ${ }^{14,15,17}$, but the morphology and number of individual cells in this network has been very difficult to define, due to this elaborate branching morphology and their relatively uniform staining with various antibody markers. Medullary TEC (mTEC) appear to be less dense, and therefore more easily defined as individual cells, but extensive heterogeneity among lineage markers has rendered the morphology of individual mTEC vague as well. Consequently, defining the size, shape, and interconnectivity of these essential cells has remained enigmatic.

Given the need for continuous $\mathrm{T}$ cell production during life, the thymus is paradoxically the most rapidly aging tissue in the body. It reaches peak tissue mass (in all species studied) prior to the onset of adolescence, and exhibits rapid and progressive atrophy afterwards, such that by mid-life most healthy mass is lost ${ }^{18,19}$. Except at very late age $\mathrm{e}^{20}$, thymic lymphocytes are essentially unchanged in the atrophied thymus, while these age related changes are primarily manifest in stromal cells, particularly cortical ${ }^{21}$. As mentioned above, niche availability provided by cTEC is the rate limiting feature for lymphoid cellularity and thymus size ${ }^{10}$. Thus, as cTEC deteriorate during aging, the thymus becomes proportionally smaller. Since new $\mathrm{T}$ cells are produced proportionally to thymic mass ${ }^{22,23}$, peripheral homeostasis thus becomes more dependent on homeostatic expansion of existing $\mathrm{T}$ cells, with the repertoire gradually drifting towards immunologic memory, with diminished broad spectrum immunity as a result ${ }^{24}$.

Remarkably, the atrophied thymus retains potent regenerative capacity, and can be induced to attain its full peak size $\mathrm{e}^{25-27}$ by stimuli such as androgen ablation. Quite logically, albeit without much evidence, thymic atrophy is assumed to result from senescence-associated cell death among TEC, while regeneration is believed to result from proliferative expansion from an epithelial stem or progenitor cell population. Consistent with these concepts, experimental loss of cTEC does result in decreased thymus size ${ }^{28}$, while induction of cTEC proliferation results in a larger thymus ${ }^{29}$. However, the fact that thymus size changes in response to TEC number (and resulting lymphoid capacity) does not mean that atrophy or regeneration, under physiologic conditions, necessarily involve changes in TEC number.

The present study stems from a large temporal database of stromal transcriptional profiles during aging and regeneration, as described later in this manuscript. Non-presumptive analysis indicates that dynamic changes in genes associated with cell size and cell morphology dominated the regeneration response. Here we use two different conditional reporter models to morphologically define and enumerate TEC in young, aged, or regenerated thymuses. Both models confirm the predictions of informatic analysis, showing that age atrophy of the thymus represents contraction of unique cell projections that characterize cTEC, while regeneration involves their regrowth. Both atrophy and regeneration occur independently of changes in cTEC number, and appear to solely reflect changes in projection morphology. Although mTEC morphology does not change appreciably with age, dynamic analysis suggests that medullary stroma may play an important role in modulation of cTEC morphology via paracrine production of known morphogens and growth factors. Our findings reconcile diverse existing concepts, and provide a revised view of atrophy and regeneration based on structural remodeling of a novel cTEC morphology that is unique among metazoan tissues.

\section{Results}

Changes in cell projection genes dominate regeneration. While thymic lymphocytes have been exhaustively studied, stromal elements that control their production have been obscured by difficulties in their isolation (the reasons for which will be made even more clear later). To circumvent this, we implemented a signal deconvolution algorithm to derive stromal transcriptomes from microdissected thymic tissue and the corresponding (purified) lymphoid components. A total of 156 microarrays are included in the present study, representing 39 medullary and 39 cortical tissue samples, and 39 medullary and 39 cortical lymphoid samples, from 5-week-old (young) mice, or 12-month-old (aged) mice before, or at various points after, castration (Fig. 1a). Regeneration data were fit to a 3rd degree polynomial spline, and in it's simplest representation, the tissue:lymphoid ratio at any point in time can be used to deconvolve the corresponding stromal transcriptome (see Fig. 1b and Methods section), thus resulting in 78 global stromal transcriptomes over time, half in each tissue compartment. For convenience, the union of stromal genes expressed at any point during the regeneration process is shown in Supplementary Data 1.

We initially focused on dynamic changes in cTEC, since this compartment dominates both atrophy and the regeneration response $\mathrm{e}^{21,30-32}$. When dynamically regulated stromal genes were mapped onto Gene Ontology $(\mathrm{GO})^{33}$ Biological Process (BP) categories (Fig. 1c), two of the five most statistically significant (i.e., over-represented) categories (empirically limited to categories with 200 genes or fewer, since very large categories always tend to be significant) were explicitly involved in cell morphology (regulation of cell shape and cell projection assembly). Two of the remaining three (positive regulation of ERK cascade and neutral lipid metabolic process) are also implicated in cell morphology 
a
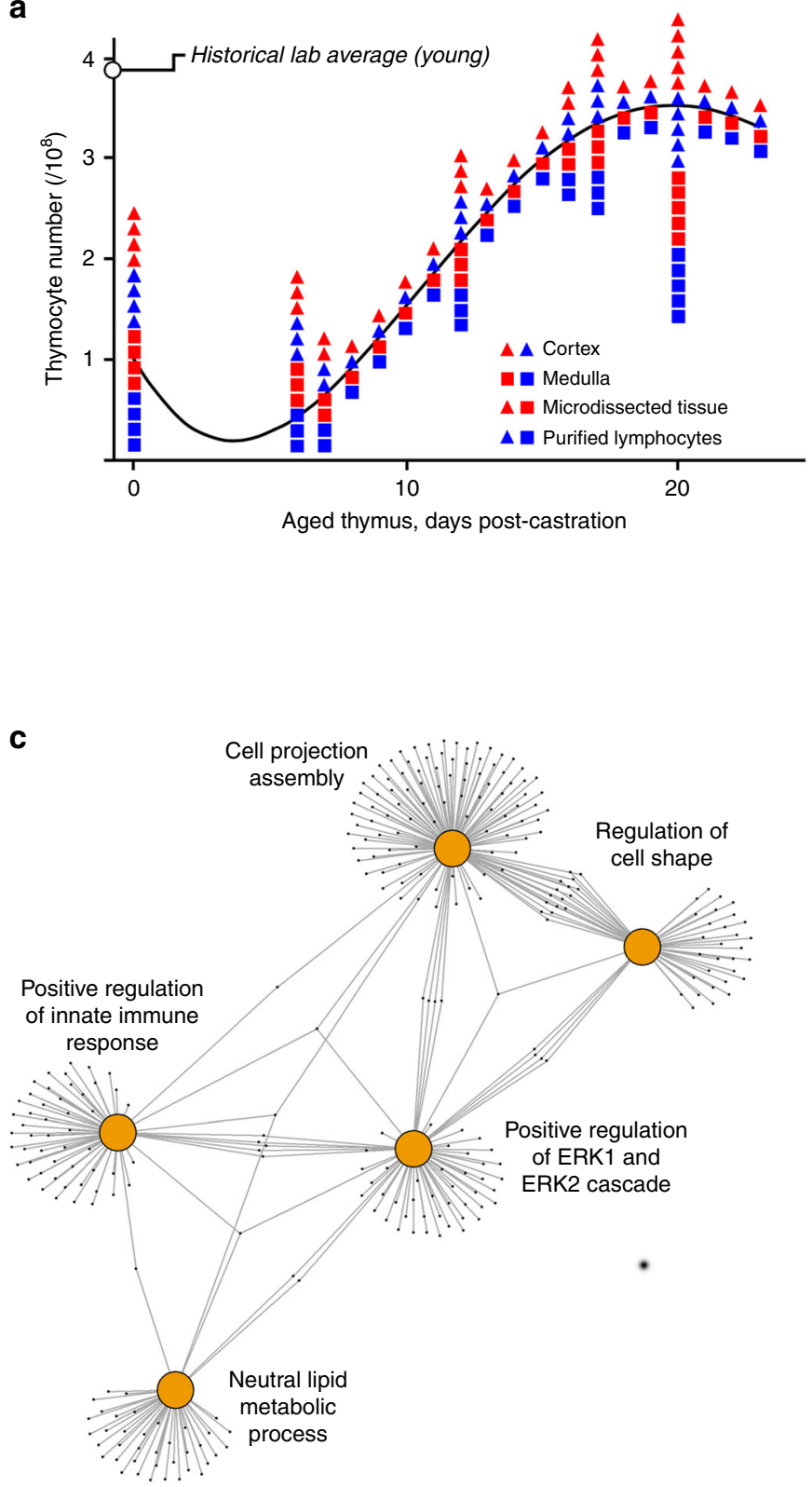

b

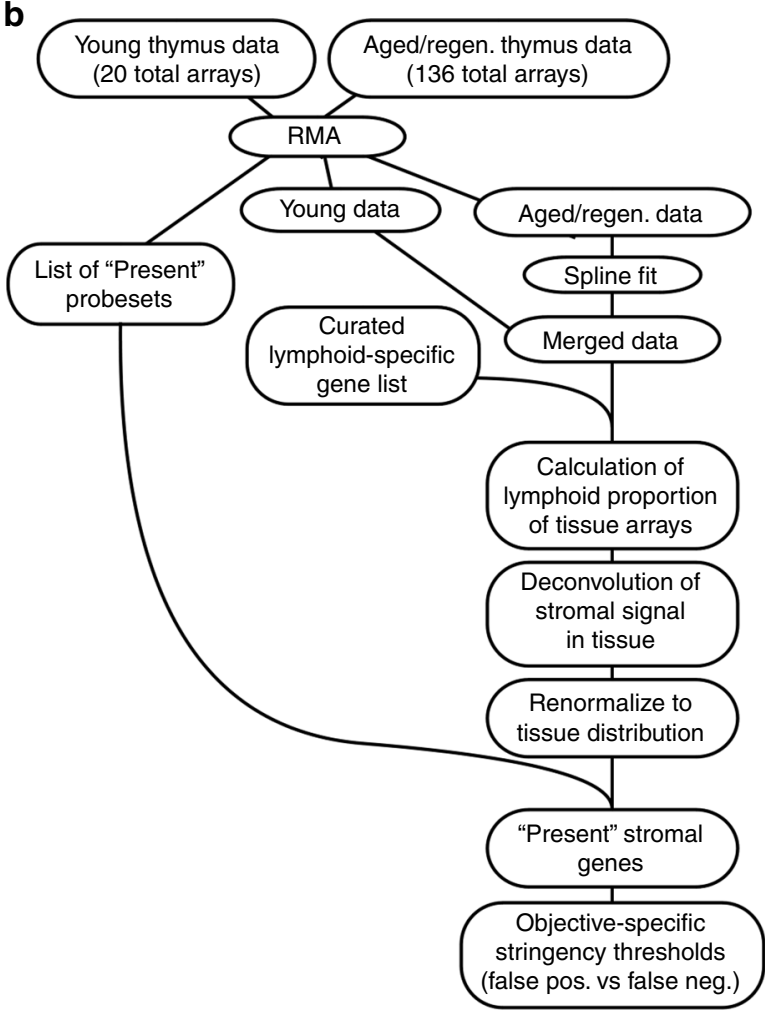

d

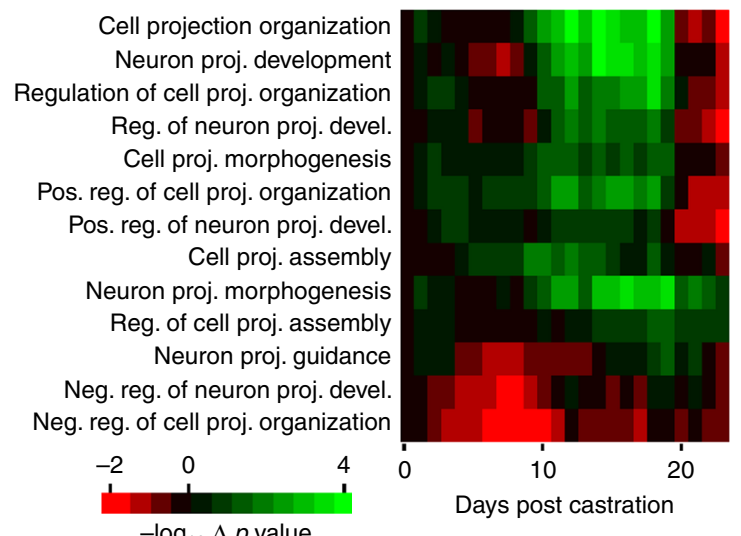

Fig. 1 Changes in cell morphology genes dominate the regeneration response. a Summary of 136 microarrays collected during castration-induced regrowth of the age atrophied thymus, mapped onto a regression curve of organ size over time (see text). An additional 20 microarrays from young thymus, 5 for each cell/tissue type, are not indicated (because they are off the time scale), representing a total of 156 microarrays used for this study. Each tissue: lymphoid pair also provides a deconvolved stromal signature (78 total) used for subsequent analysis. b General workflow of steps in the deconvolution of stromal gene expression. c A network map of the top 5 GO:BP categories (200 genes or fewer) indicates a strong bias in cortical stroma towards dynamically regulated genes associated with cell projection morphology. Connections between hubs indicate shared genes. $\mathbf{d}$ Mapping of deconvolved thymic stromal transcriptomes onto GO categories related to cell projection (and containing at least 10 total genes). The heat map shows the change in statistical significance (i.e., change in Fisher exact test $p$ value) over time of regeneration, normalized to day 0 . There is a negative enrichment of negative regulators early, and a positive enrichment of positive regulators late, further supporting the prediction from non-presumptive analysis that changes in cell shape may play a significant role in the regeneration response

and cell membrane projections, just not as exclusively as the others. Not surprisingly, then, dynamically regulated genes in the stromal response (Supplementary Data 1) are extensively populated by genes associated with cell adhesion, guidance response, membrane proximal signaling, and cytoskeleton remodeling. These observations, together with the known branching morphology of TEC (especially cTEC), prompted us to look further into this enrichment. GO BP ontologies containing the term projection, and the genes associated with them, were identified, and those ontologies containing at least 10 members were extracted. For each, over-representation of genes in the stromal list at each time point was tested using the Fisher exact test (corrected for false discovery rate, all genes as background). The results are shown in Fig. 1d, normalized to day 0 (12 months of age, pre-castration). Remarkably, all projection BP exhibited significant regulation of cell projection genes, with two main patterns found, namely, depletion of negative regulators early followed by restoration later, or enrichment of positive regulators 
early followed by their depletion later. These patterns align closely with the regeneration kinetics we have previously described (Fig. 1a, and ref. ${ }^{21}$ ), and prompted us to speculate that changes in stromal morphology, rather than changes in stromal cell number, might explain thymic atrophy and regeneration.

Genetic labeling reveals a unique morphology for cTEC. Based on standard immune marker staining, cTEC are known to possess extensively branched cell processes ${ }^{14,15,17}$ but conventional staining cannot distinguish where one cell ends and the next begins, or the size or shape of individual cells. To fill this void, we utilized the Confetti (Brainbow2.1) conditional allele ${ }^{34}$, wherein the Rosa26 locus has been modified to include a floxed stop codon upstream of two cassettes of two fluorescent proteins in inverted transcriptional orientation, with each cassette flanked by inverted loxP sites. In the presence of Cre, the stop codon is deleted and one of the four fluorescent proteins is placed under the transcriptional control of Rosa26. The four fluorescent proteins are a membrane-targeted cyan fluorescent protein (mCfp), a nuclear targeted green fluorescent protein (nGfp), and cytoplasmically retained yellow and red fluorescent proteins (cYfp, cRfp). nGfp is not useful for determining TEC morphology, since it labels only the nucleus. Reporter activation in virtually all TEC (see Supplementary Fig. 1) was induced via an allele containing a bicistronic Cre knockin of the Foxn1 locus ${ }^{35}$. Images from such thymuses are shown in Fig. 2. A wide area view (50 $\mu \mathrm{m}$ maximum $\mathrm{Z}$ projection) of tissue from a young adult mouse thymus ( 5 weeks) is shown in Fig. 2a, revealing randomly colored TEC in a variety of hues (note: as long as Cre is expressed, the Confetti allele can continue to rearrange, and thus intermediate mixed hues can be seen). A 3D axial video of this same projection volume is shown in Supplementary Movie 1. A higher magnification of a cortical region is shown in Fig. 2b. The ability of the Confetti allele to define individual cells where conventional cTEC markers cannot (in this case, keratin-8) is highlighted by Fig. 2c. Viewed in 3D (see orthogonal projections in Fig. 2d, or the associated animation in Supplementary Movie 2), individual cTEC exhibit a morphology not seen in any other cell or tissue type. Consistent with conventional immunostaining, they exhibit an extensive network of projections. However, these projections are not branches in the traditional sense, i.e., progressively thinning bifurcations resulting in terminal ends, like a neuron or dendritic cell. Instead, cTEC cell projections form of long, looping structures that, in turn, generate an extensive intracellular labyrinth of 20-30 voids in each cTEC. Each void, in turn, contains approximately 2-10 cortical lymphoid cells (as determined by DAPI or CD90 staining, see Fig. 2e), totaling about 100-150 lymphoid cells almost totally engulfed by each cTEC. Connections between adjacent cTEC occur mainly through abutment of opposing loops (Fig. 2f, and Supplementary Movie 3), rather than via synapsed terminal ends (as in neurons, for instance), although some connections via synapses are also seen. Although the fine structure of individual loops is variable, overall shape, size, and orientation in cTEC is fairly consistent (best visualized with young thymus in Supplementary Movie 1), representing a slightly flattened ovoid (see Fig. 2d) approximately $75 \pm 10 \mu \mathrm{m}$ in its long dimension, which is radially aligned towards the capsule. Long axis radial alignment in cTEC, was first proposed over 30 years ago $^{15}$, and shows that like other epithelial types, cTEC are polarized, although it mostly obscured by their complex morphology and deep embedding in lymphocytes.

In the 12-month-old thymus, which is about $25 \%$ of the volume/cellularity found in the young thymus ${ }^{21}$, dramatic differences are seen (Fig. 2g-i). Pigment bodies with broadspectrum fluorescence emission ${ }^{36}$ are conspicuous (Fig. $2 \mathrm{~g}, \mathrm{~h}$ ), and not found in young (see Supplementary Fig. 2), These autofluorescent inclusions, which represent classic hallmarks of aging 37,38 , initially presented an obstacle to morphometric analysis. However, we noted that by using $405 \mathrm{~nm}$ excitation with a $570-610 \mathrm{~nm}$ collection window, we could independently image autofluorescence, and thus remove it from reporter images (documented in Supplementary Fig. 2). Confetti labeling in aged cTEC was conspicuously less robust than that seen in young (Fig. $2 \mathrm{i}$ vs. $2 \mathrm{a}$ ). In many regions it was difficult to detect cTEC cell processes without ultra high magnification, but they do remain pervasive throughout the atrophied cortex in this diminished condition. Changes in cTEC cell morphology are most pronounced in the sub-capsular space (Fig. 2i), which notably represents the region where the highest density of ageassociated pigments is seen (Fig. 2h). Various morphological parameters, such as total surface area, feret (maximum) diameter, or occupied cell volume (Fig. $2 \mathrm{k}-\mathrm{m}$ ) quantitatively confirm contraction of cTEC projections with age. In this context, we wish to emphasize that our measurements must underestimate the real difference, since the worst affected cTEC were so difficult to distinguish from background that they could not be measured. Nonetheless, quantitative, as well as qualitative findings show that aging is associated with a very significant diminution in cTEC morphology, represented by a contraction in projection length/complexity as well diameter.

To determine whether thymic regeneration correlated with rejuvenation of cTEC morphology, we analyzed aged Confetti thymuses 21 days after surgical castration, representing peak regenerated size ${ }^{21}$. In general, cTEC morphology at this time was greatly enhanced, although the cortex was not identical to young (Fig. 2 j vs. $2 a)^{21}$, despite similar size. Instead, cTEC morphology in the regenerated thymus was widely variable, with the conspicuous appearance of regions of unusually large cTEC, as well as other regions where cTEC morphology remained poor, qualitatively (Fig. 2j) or quantitatively (Fig. $2 \mathrm{k}-\mathrm{m}$ ). The reason for this non-uniformity is difficult to pin down, but might reflect cells that were more or less damaged by aging. Again, our quantitative analysis (Fig. 2k-m) probably underestimates the frequency of very large cells, since they are more likely to exceed the boundaries of any fixed optical volume, and thus cannot be measured. Consequently, the quantitative measurements shown in Fig. $2 \mathrm{k}-\mathrm{m}$ are conservative, but nonetheless confirm reextension after regeneration. It is worth noting that age associated autofluorescent bodies remain a prominent feature in the fully regenerated thymus (see Supplementary Fig. 2), further emphasizing our previous finding that even though cellularity of the thymus may be temporarily restored by castration, the underlying consequences of aging are permanent ${ }^{21}$.

mTEC morphology is unchanged during atrophy and regeneration. Our previous work showed that atrophy and regeneration are primarily functions of changes in cTEC, while the medulla is minimally affected ${ }^{21}$, consistent with the findings of others $^{30-32}$. However, it is possible that there could be qualitative changes in mTEC that do not result in changes in medullary volume. In any case, like cTEC, the morphology of individual mTEC is poorly defined. To accomplish this, we used the cYfp and cRfp choices in Confetti, which produce filled objects (cells) that can be readily segmented and characterized. To define ideal parameters defining individual mTEC, we generated mice carrying one Rosa26[Confetti] allele and one Rosa26 allele bearing a conditionally stopped histone $2 \mathrm{~b}(\mathrm{H} 2 \mathrm{~b})$ :mCherry fusion protein $^{39}$, resulting in red fluorescent labeling of all TEC nuclei after induction of both alleles by Foxn1[Cre]. This allowed us to train the segmentation approach (see Methods) until there was 

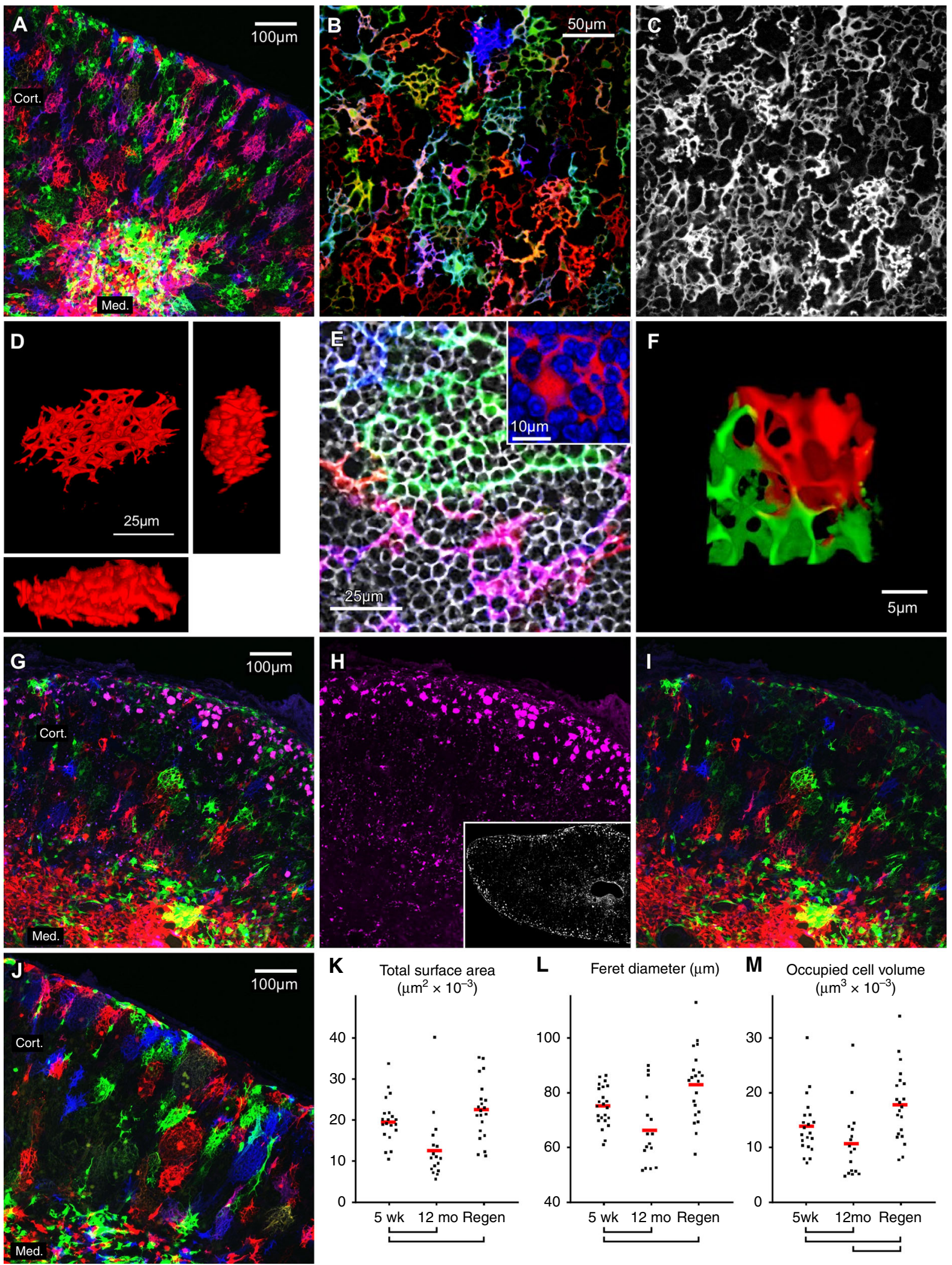

reasonable certainty $(>95 \%)$ that we were defining individual mTEC containing a single nucleus. This approach was then applied to cYfp and cRfp image stacks from both young and aged Confetti mice. As shown in Fig. 3 and Supplementary Movies 46 , Confetti revealed a diverse range of mTEC morphologies, in contrast to cTEC, which had essentially a single canonical shape. These diverse mTEC morphologies did not fall into identifiable subtypes, but rather formed a continuous distribution (Fig. 3d-g), even when other morphological parameters were simultaneously tested for clustering. Using three highly discriminatory parameters (feret diameter, the largest possible linear distance between two points of an object; compactness, the ratio of the surface area of an object to its volume; or volume:ellipsoid ratio, the ratio of the volume of an object to the volume of the smallest 
Fig. 2 cTEC exhibit a unique morphology that fluctuates during aging and regeneration. Data are from Foxn1[Cre $]^{+}$Rosa26[Confetti] + thymuses. Blue $=\mathrm{Cfp}$, green $=$ Yfp, red $=$ Rfp. Mouse age was: a-f, 5 weeks, $\mathbf{g}-\mathbf{i}, 12$ months, and $\mathbf{j}$ is peak regenerated. Comparable panels (e.g., $\mathbf{a}, \mathbf{i}, \mathbf{j}$ ) were processed identically. 3D renderings can be found in Supplementary Movies 2-4. a Maximum Z projection of a $40 \mu \mathrm{m}$ optical stack (the approximate transverse depth of a single CTEC) at 5 weeks. b Zoomed view of the cortex ( $6 \mu \mathrm{m} Z$ projection), revealing distinctions between individual cells, in contrast to $\mathbf{c}$, which is conventional CTEC marker expression (Krt8) in the same stack. Scale bar in $\mathbf{b}$ also applies to $\mathbf{c}$. $\mathbf{d}$ Orthogonal views of a typical cTEC, revealing looping structures forming a complex labyrinth of intracellular voids filled by lymphoid cells (e), as indicated by Thy-1 staining (gray) or DAPI staining (inset, blue). $\mathbf{f} 2 \mathrm{D}$ representation of the interface between two CTEC, showing that most contact occurs via abutment of looping structures, rather than synapses. $\mathbf{g}$ Maximum $Z$ projection of a $40 \mu \mathrm{m}$-thick optical stack from a 12-month-old mouse. Confetti detection is obscured by autofluorescent pigments associated with aging, but these can be imaged independently (h), and thus subtracted (i; see also Supplementary Fig. 2). Scale bar in $\mathbf{g}$ also applies to $\mathbf{h}$ and $\mathbf{i}$. Inset in $\mathbf{h}$ is at $20 \times$ magnification. cTEC in the aged thymus (i) are much less conspicuous than in young (a), reflecting both contraction of total cell dimensions ( $\mathbf{k}-\mathbf{m})$ and thinning of the remaining projections. $\mathbf{j} 40 \mu \mathrm{m} \mathrm{Z} \mathrm{projection} \mathrm{from} \mathrm{a} \mathrm{12-month-old} \mathrm{mouse} \mathrm{on} \mathrm{the} \mathrm{peak} \mathrm{of} \mathrm{regeneration} \mathrm{(day} \mathrm{20).} \mathrm{Confetti} \mathrm{intensity} \mathrm{is} \mathrm{much}$ more robust than in the aged thymus (j), but is not restored to the status of the young (a), with some cells retaining an aged phenotype (atrophic), while others are abnormally large. $\mathbf{k}-\mathbf{m}$ measurement of relevant physical parameters in cTEC, such as total surface area, feret diameter, or cell volume. Brackets indicate statistical significance (two-tailed $t$ test, $p$ value $<0.05$ ). $n=3$ biologically independent tissues each for 5 weeks, 12 months, and regenerated groups. Source data are provided in the accompanying Source Data File
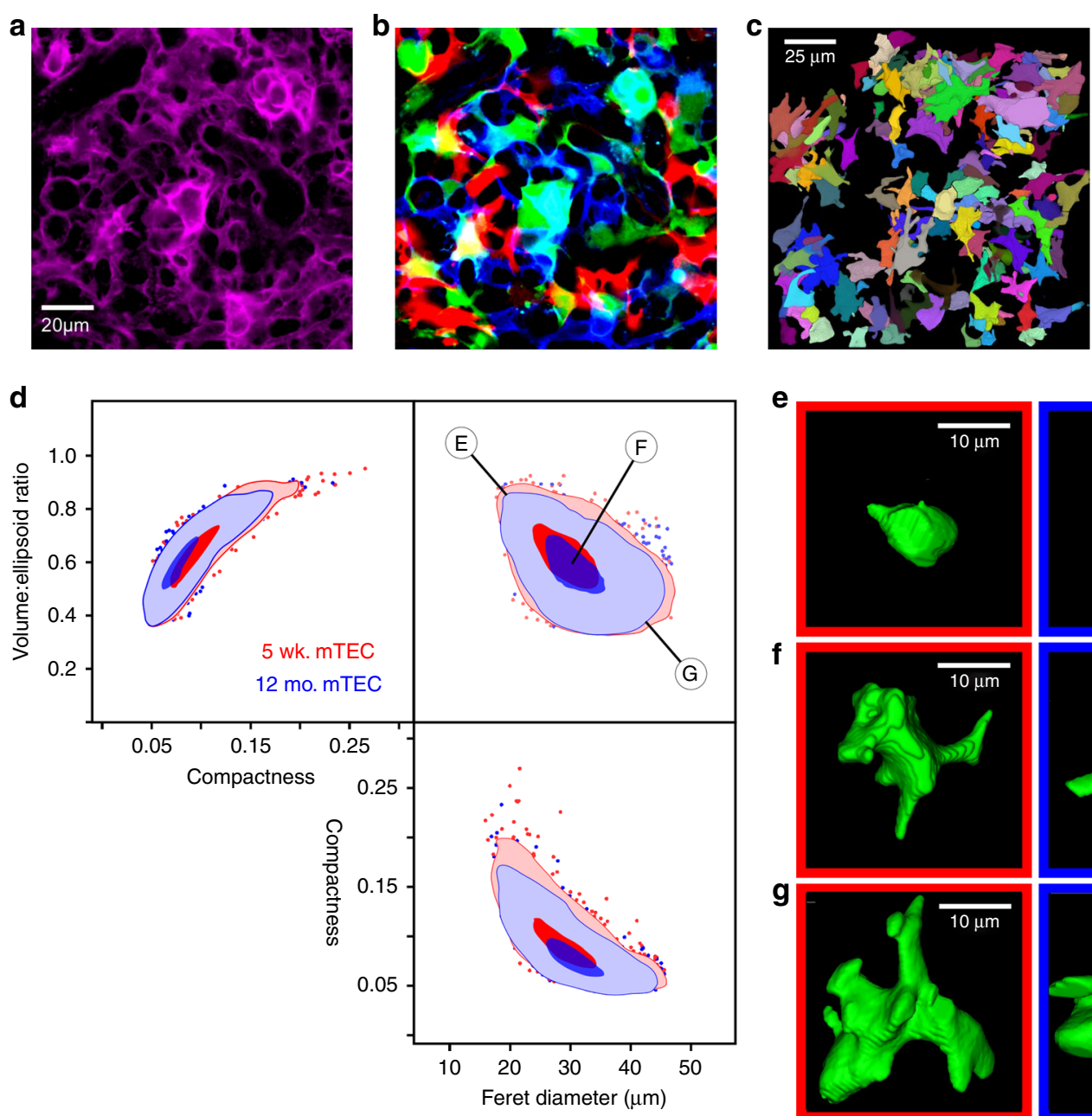

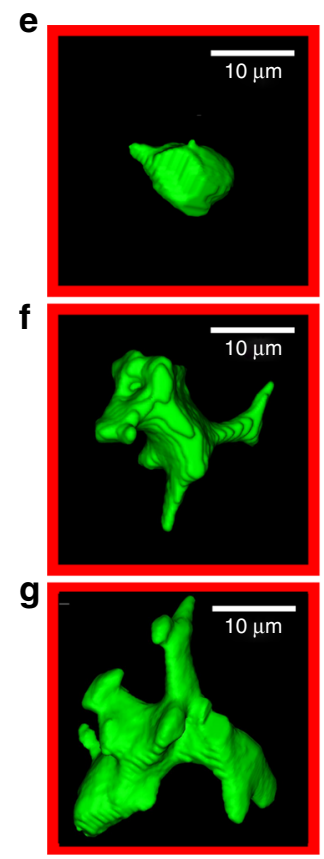

5 wk. mTEC

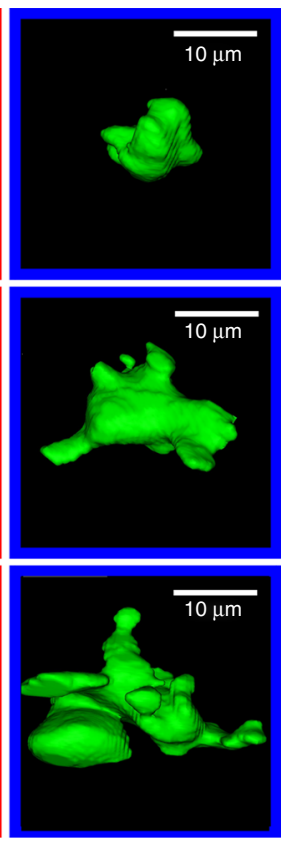

12 mo. mTEC

Fig. 3 mTEC exhibit a broad spectrum of shapes that change little during atrophy. a A maximum Z projection ( $2.5 \mu \mathrm{m}$ thickness) of medulla from a young ( 5 weeks) Confetti thymus, stained with a conventional mTEC marker (Epcam). Note that individual cells cannot be distinguished. $\mathbf{b}$ Confetti fluorescent proteins ( $\mathrm{mCfp}, \mathrm{cYfp}$, and $\mathrm{cRfp}$ ) in the same tissue volume, showing clear distinction of individual cells and their shapes. Scale bar in a also applies to b. c 3D projection of a $25 \mu \mathrm{m}$-thick stack of one color channel (cYfp) from the young Confetti medulla, with individual cells labeled by a random palette. d Orthogonal projections of the 3D distribution of mTEC shapes from young (red) or old (blue) mice. At least 1000 objects are shown for each age. Dark colors indicate $10 \%$ probability limits, light colors show $90 \%$ probability limits, and the remaining events are shown as individual dots. An overlapping (between young and old) unimodal distribution is found, but with a broad diversity of shapes $(\mathbf{e}-\mathbf{g})$, ranging from larger and extensively branched to smaller and non-branched morphologies 
circumscribing ellipsoid), the shape distributions of young and aged mTEC were virtually indistinguishable (Fig. 3d), further emphasizing that the primary target of accelerated age atrophy in the thymus is cTEC and the cortex, while mTEC and the medulla remain essentially undisturbed.

TEC numbers remain stable during atrophy and regeneration. Our lab and others have shown that thymic atrophy is primarily a function of decreased cortical volume $e^{21,30-32}$ resulting from age related changes in $\mathrm{cTEC}^{21}$. Confetti labeling (Fig. 2) shows that aging involves contraction of the extensive cell projections that characterize cTEC, and thus of overall cTEC size. Diminished size of individual cTEC, in the absence of cell death, should lead to an increase in the apparent density of cTEC. To test this, we used the Rosa26[H2b:mCherry] allele described above ${ }^{39}$, activated by Foxn1[Cre], to unambiguously label the nucleus of all TEC. Results are summarized in Fig. 4. Consistent with our predictions, aging resulted in a very significant increase in the density of TEC in the cortex, but not the medulla, both visually (Fig. $4 \mathrm{a}-\mathrm{d}$ ) and quantitatively (Fig. 4e, f). Consistent with a dominant role for the outer cortex in atrophy, the highest density of cTEC is seen in the outer cortex and sub-capsular region. Note that the capsule and the immediate sub-capsular region were actually excluded from quantitative density measurements, since their distribution approximates a curved $2 \mathrm{D}$ area density rather than a $3 \mathrm{D}$ volume density. Thus, the quantitative results presented for cortical density with age (Fig. 4) again underestimate the true extent of this process. Together with Confetti marking, these cell number/ cell density experiments confirm that age related atrophy of the thymus is explained by contraction of cortical volume due to shrinkage of cTEC in the absence of cell death, resulting in an apparent increase in their density. Our findings further indicate that cortical atrophy initiates in the outer cortex/subcapsular region (also coincident with the highest density of age-associated pigment inclusions), and gradually progresses inward.

Relevant to our estimates of TEC number, a recent study used a combination of conventional staining and reporter labeling to quantitate TEC in the young thymus ${ }^{40}$, and concluded that mechanical/enzymatic methods for isolating TEC from the thymus grossly underestimate the total number of TEC found in situ. They conclude that many TEC either are not efficiently released by these methods, or they are destroyed in the process. Our results strongly support this, and the genetic labeling approach we used, which has the advantage of labeling only TEC while ignoring everything else, suggests that the number of TEC is even higher. Our measured densities (Fig. 4) applied to the tissue volumes estimated by those authors suggest that cTEC number in the young thymus is about 3 -fold higher than their measurements $\left(9.9 \times 10^{5}\right.$ cTEC per lobe, vs. $\left.3.7 \times 10^{5}\right)$, and 5 -fold higher for mTEC $\left(2.6 \times 10^{6}\right.$ total, vs. $\left.4.9 \times 10^{5}\right)$. Our findings are very consistent with an earlier electron microscopy study ${ }^{41}$, which showed $6.8 \times 10^{5}$ cTEC and $2.6 \times 10^{6}$ mTEC per thymus at 4 weeks of age. Regardless of any differences between these studies, all of them reinforce the conclusion that TEC are much more numerous than predicted by the yields from physical isolation. This suggests that methods for removing TEC from the thymus yield a minor proportion of the total cells, which may or may not be representative of the remainder or the group as a whole. Not surprisingly, for reasons related to their complex and fragile morphology, this discrepancy is greatest among cTEC, which are apparently isolated with a very low success rate (see Discussion).

Regeneration does not require changes in TEC proliferation. To this point, our data support the conclusion that age-related atrophy of the cortex results from contraction of cTEC projection morphology, rather than by senescence-associated death of cTEC. The corollary is that regeneration can be explained primarily by the re-extension of cTEC cell projections, independent of proliferation. Several laboratories have addressed proliferation in mTEC using in vivo DNA labeling ${ }^{42-44}$, but always by analyzing isolated cells, which, as described above, is now known to dramatically underestimate the total population, and may be skewed towards certain cell types. As a further complication, labeling was always performed for multiple hours to days, which can result in production of labeled non-mitotic daughter cells (i.e., one mitotic precursor producing two post-mitotic progeny), thus obscuring the true proliferative index. Thus, a clear static assessment of mTEC proliferation in situ is lacking. Among cTEC, the situation is even less clear. One study suggested that castration restored the mTEC/cTEC ratio ${ }^{45}$, but data were not shown. Another study ${ }^{46}$ indicated that IL-22 can increase cTEC proliferation, but basal levels in untreated mice were again not shown, and mechanically isolated cells were used. Thus, the proliferative status of cTEC in the young, aged, and regenerated thymus remains unresolved.

To address this, we used the thymidine analog 5-ethnyl- $2^{\prime}$ deoxyuridine (EdU) to efficiently label cells in S phase in Foxn1 [Cre]/H2b:mCherry mice in an acute $(60 \mathrm{~min})$ in vivo pulse, followed by copper-catalyzed detection ${ }^{47}$ using a fluorescently tagged alkyne (ThermoFisher \# C10337). Thymus sections were taken from young (5-week-old) mice, as well as from 12-monthold mice prior to castration or at mid-phase of the regeneration response (day 13 most-castration, see Fig. 1a and (day 13 postcastration, see Fig. 1a and ${ }^{21}$ ), where proliferation of any cell should be present at a maximal rate. Intensely proliferating lymphocyte progenitors conveniently served as an internal control for EdU penetrance, and were brightly labeled throughout the thymus (Fig. 5a-c), with very high density in the outer cortex, as previously described ${ }^{8,48}$. Labeled mTEC were readily detected at all ages (Fig. $5 \mathrm{~d}-\mathrm{f}$ ). In the young thymus, approximately $4.5 \%$ of all mTEC were in $\mathrm{S}$ phase (Fig. $5 \mathrm{~g}$ ), which, in general, is consistent with values obtained using different methods and labeling times ${ }^{44,45}$. Also consistent with previously published work ${ }^{45}$, we find that proliferation of mTEC is reduced approximately threefold with age (Fig. 5g). However, even with a large number of unambiguously defined mTEC being counted, we found no change in $\mathrm{mTEC}$ proliferation during regeneration (Fig. 5g), consistent with a minimal role for the medulla in this process $^{21}$.

In contrast to mTEC, changes in cTEC are a dominant factor in age related atrophy and regeneration (Figs. 2 and 4, and ref. $\left.{ }^{21}\right)$. Consequently, if proliferation of cTEC were a major mechanism for regeneration of the thymus after castration, one would expect to see a conspicuous increase in EdU-labeled cTEC after castration. However, as shown in Fig. 5h-k, this was not the case. Proliferating cTEC were very rare or non-existent in the cortex, even in the young thymus, where a single EdU-labeled cTEC was found among dozens of image volumes representing thousands of cTEC (note: the image volumes described for Fig. 5 were formally quantitated, but many more were manually examined without formal measurement). In the aged or regenerating thymus, no labeled cTEC could be found at all. It might be tempting to contemplate a technical error, except that lymphocyte labeling in the same regions is conspicuous (Fig. $5 \mathrm{~h}-\mathrm{j}$, green only), and unlabeled (by EdU) cTEC nuclei are also readily visible (red only). Consequently, we conclude that proliferation among cTEC is very limited in the steady-state young thymus, and does not increase during regrowth. Consistent with EdU labeling, genes associated with cell cycle do not change significantly during castration induced regrowth (Fig. 5l), with the exception of a modest change in cyclin B1, which may be 

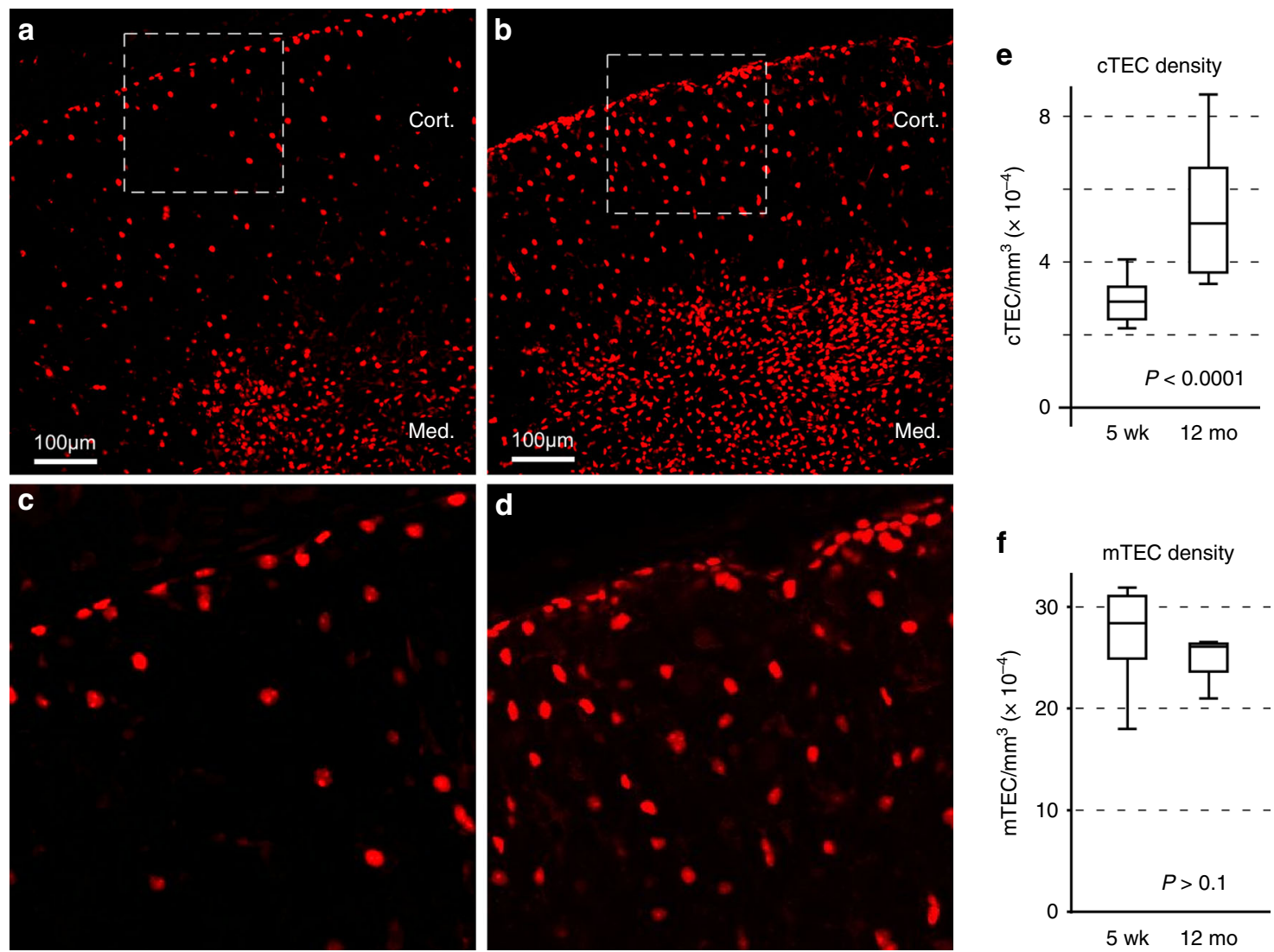

Fig. 4 CTEC density increases with atrophy, indicating contraction of cell size without changing in number. $\mathbf{a}$, $\mathbf{b}$ Wide area view of thymus from 5 week or 12 -month-old mice (respectively) in which an $\mathrm{H} 2 \mathrm{~b}$ :mCherry fusion protein is conditionally activated in all cells of the TEC lineage. Images represent a maximum Z projection of an optical slice with thickness equal to the average feret diameter of one TEC nuclei ( $11 \mu \mathrm{m})$. Paired images were acquired and processed identically. Regions indicated by dashed lines are shown in panels $\mathbf{c}$ and $\mathbf{d}$. Consistent with this visual assessment and the contraction of cTEC projections by the Confetti reporter, measurement of TEC density shows that CTEC density increases dramatically with age, while mTEC density is not significantly changed (e and $\mathbf{f}$, respectively; statistical significance calculated by two-tailed $t$ test). Data indicate total nuclei per image volume from at least 3 independent thymuses of each type, with each thymus represented by one or more distinct image volumes until large numbers of nuclei were counted. Total events counted were 5060 (young cortex), 3567 (aged cortex), 17,097 (young medulla), or 10,176 (aged medulla). Boxes represent the interquartile range with the median indicated by a horizontal line; whiskers indicate the full range. Source data are provided in the accompanying Source Data File

playing a non-cell cycle related role, or may be spurious, but in any case cannot act alone to promote cell division. Together, data from Confetti and EdU labeling experiments confirm that the non-presumptive predictions made by our temporal gene expression database (Fig. 1) are correct, and that cortical atrophy and regeneration are controlled by modulating the extensive cell projections found in young cTEC, rather than a change in CTEC number.

Trans-acting medullary signals may regulate cTEC morphology. Our database of stromal and lymphoid gene expression during aging and regeneration allowed us to predict that the size and shape of cTEC, rather than changes in their number, were the driving force behind atrophy and regrowth. While regulation of cell size and shape are highly complex and multidimensional processes not amenable to rapid dissection, the same database, together with existing knowledge of critical regulators of cell size and shape in many developing organisms, allows us to make mechanistic predictions about this process for further study. We used the enrichPathway function of the ReactomePA package in $\mathrm{R}$ to define Reactome pathways (reactome.org) that were significantly enriched in cortical stroma (see Methods). These were then limited to pathways with $\leq 200$ genes associated with the keywords signaling or signaling (spelling variants). The ten most significant of these pathways are shown in Fig. 6a; 8 of these 10 (excluding IL1 and Clec7A) have obvious strong ties to cell size, shape, and epithelial remodeling. Note that 3 of the 4 most significant pathways are associated with Mtor, which is widely recognized as a critical regulator of cell and tissue size via stimulation of energy metabolism, protein and lipid synthesis, and cytoskeletal reorganization ${ }^{49,50}$. Notably, Mtor is also recognized as a high priority target for aging intervention and increased longevity ${ }^{51}$, and inhibition of Mtor activity (via treatment with rapamycin) has been shown to diminish thymus size $\mathrm{e}^{52}$. Further strengthening these associations, the significance of the Mtor pathway in cortical stroma changes dramatically with aging and regeneration (Fig. 6b), with relatively high representation in the young or during regrowth, and diminished representation in the aged or in terminal regeneration. Analysis of the individual Mtor pathway genes expressed by cortical stroma also reveals dynamic regulation of many pathway components (Fig. 6c), including the key regulatory component Tscl.

Soluble ligands targeting the Mtor pathway, and more broadly, targeting most classical pathways associated with cell size and shape (e.g., Wnt, Tgfb, etc), were generally not expressed or were not dynamically regulated by cortical stroma. Many of these could possibly be endocrine in origin, although it is important to remember that the thymic cortex (but not the medulla) is immunologically privileged, and is protected by a nearly 

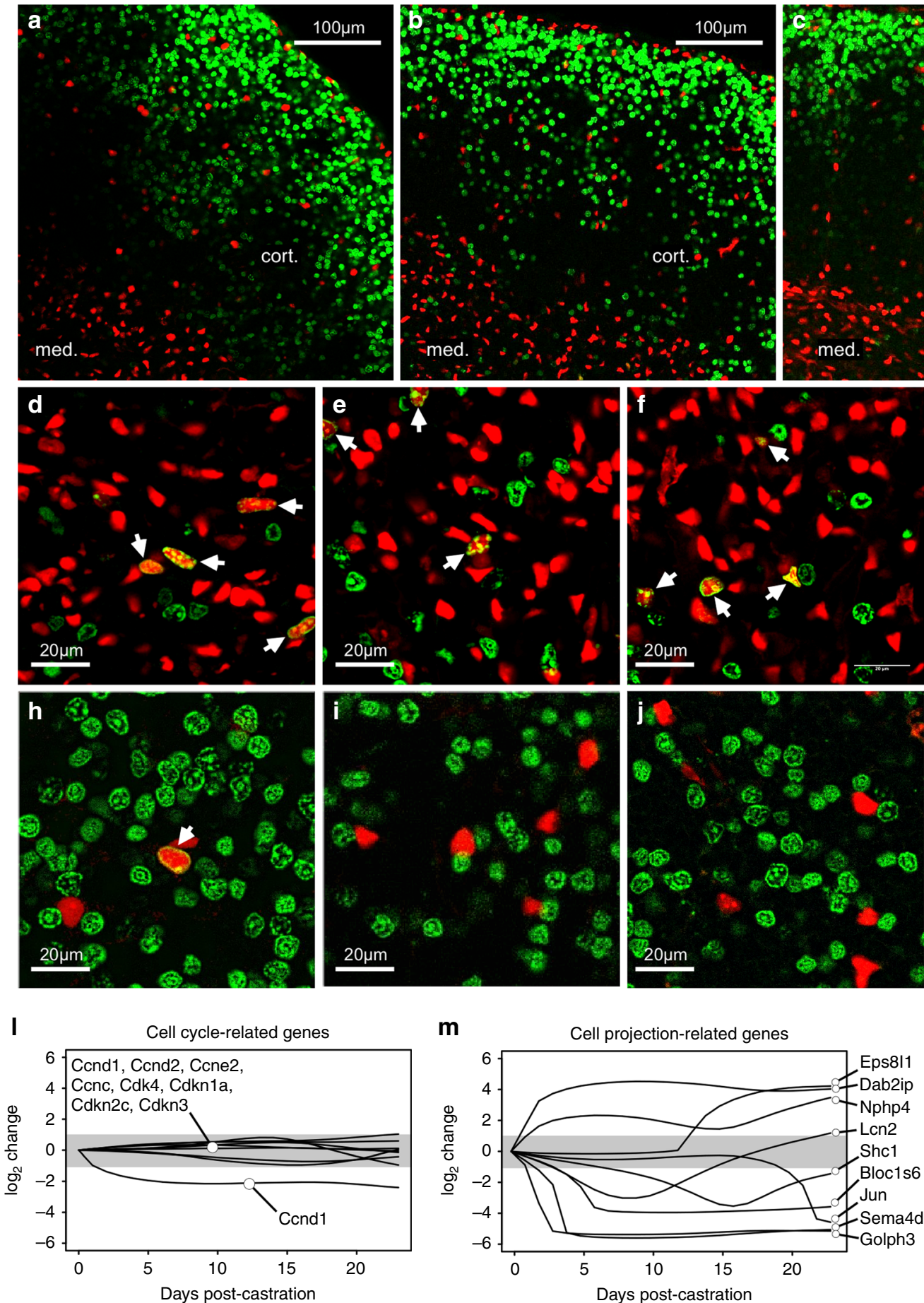
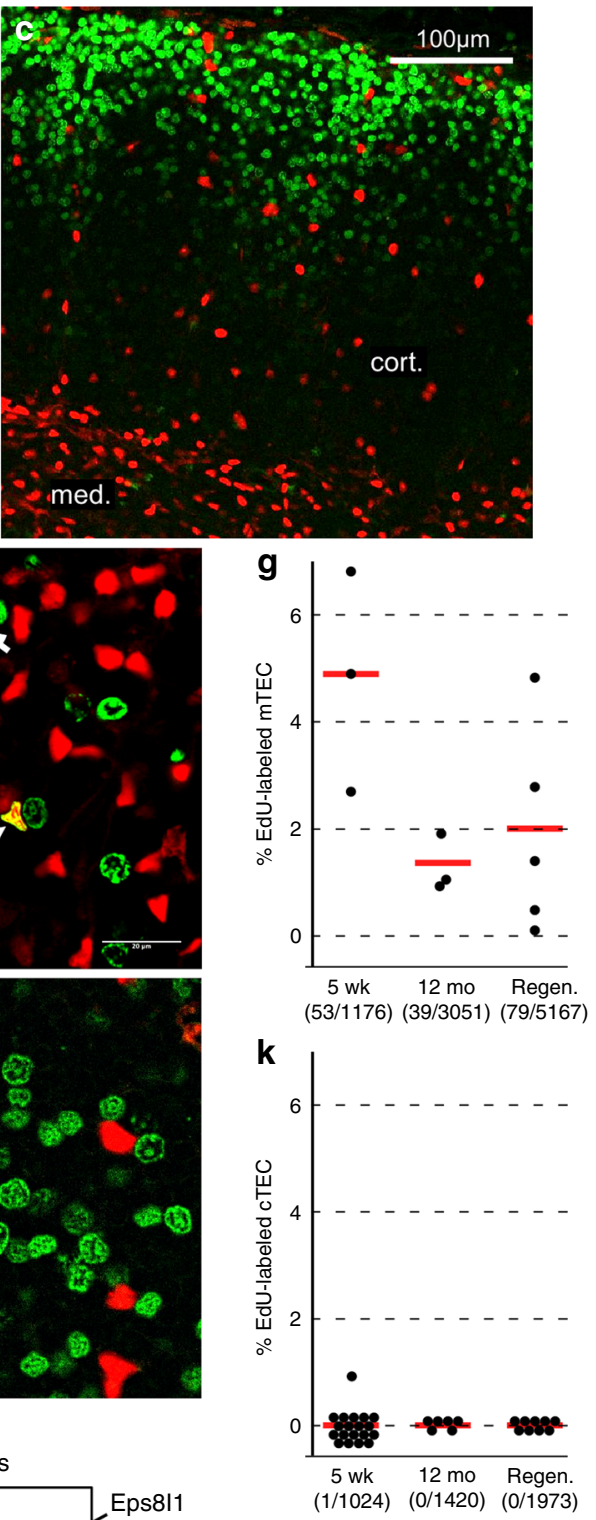

Fig. 5 Regeneration of the cortex occurs independently of CTEC proliferation. a Wide area view of a single optical plane of thymus from a 5-week-old mouse in which $\mathrm{H} 2 \mathrm{~b}$ :mCherry (red) was conditionally activated in TEC by Foxn1[Cre], $1 \mathrm{~h}$ after a single injection of the thymidine analog 5-ethnyl-2'-deoxyuridine (EdU, green). $\mathrm{EdU}^{+}$nuclei are not obvious in the deeper tissue because display levels are limited by the intense staining of cortical lymphoblasts, but they are nonetheless found throughout the organ. $\mathbf{b}$, c Similar wide area views from 12 month thymuses, or thymuses from 12-month mice at the midpoint of regrowth (day 14 post-castration), respectively. d-f Higher magnification views of the medulla in thymus from 5 weeks, 12 months, or regeneration day 14 . Edu ${ }^{+}$mTEC nuclei are readily detectable (arrows) in all tissues, although the frequency does decrease with age ( $\mathbf{g}$ ), a feature that does not change after regeneration. Data points represent the percentage of labeled nuclei in individual image volumes from three biologically independent thymuses for each sample type (young, aged, regenerated). Red bars indicate the mean of these percentages. Numbers below sample types indicate pooled EdU+ nuclei/pooled total nuclei for each sample type. $\mathbf{h}-\mathbf{j}$ Similar analysis of the cortex in the same order of appearance. A single Edu+ cTEC was found out of 1024 nuclei that were formally counted in multiple volumes from at least 3 tissues) (h, k); many more volumes were examined (but not formally counted) without finding additional labeled cells. No labeled nuclei were found in CTEC from 12 months or regenerating thymus showing that tissue regeneration does not correlate with an increase in cTEC proliferation. I Consistent with the near absence EdU labeling in CTEC, genes associated with cell cycle do not fluctuate during castration-induced regeneration; only one of these (cyclin b1) exhibits a 2-fold or greater change (indicated in gray) during induced regrowth. $\mathbf{m}$ For comparison, an equal number of dynamically regulated genes found within the cell projection ontologies (see Fig. 1). Source data are provided in the accompanying Source Data File 
impermeable blood-thymus barrier ${ }^{53}$. However, we find that medullary stroma is enriched in, and dynamically regulates, multiple ligands for Mtor and other morphogenic pathways (Fig. 6d), including recognizable TEC signals such as $\operatorname{Igf1} 1^{54}$ or Fgf $21^{55}$. Our data thus suggest that multiple pathways regulating cell size and shape in other tissues also control the branching morphology of cTEC, with a dominant role for Mtor. Further, it appears that induction of these pathways in CTEC may be under the dynamic control of paracrine factors produced by medullary stroma, which is apparently responding to systemic endocrine signals induced by castration. Overall, our findings are most consistent with a mechanism in which cortical atrophy represents progressive degeneration of cTEC morphology due to diminished production of paracrine signals from the medulla, while castration-induced regeneration represents a transient replenishment of these signals, and the corresponding restoral of cTEC morphology and cortical volume.

\section{Discussion}

Since $\mathrm{T}$ cells must be continuously produced throughout life, the accelerated atrophy of the thymus with age is enigmatic, especially given its latent regenerative potential. Age-associated atrophy is common to many tissues (for instance, muscle, CNS, skin, and testes), and can be a consequence of cell loss (death), but often is associated with the shrinkage (atrophy) of individual cells, collectively resulting in tissue atrophy. Likewise, regeneration may be attributed to proliferation of stem or end stage cells (hyperplasia), but can also result from growth of existing cells (hypertrophy) that is independent of proliferation. Despite the magnitude of atrophy and regeneration in the thymus, the underlying mechanisms for these processes have remained obscure, although our previous findings show that both are attributable to changes in $\mathrm{CTEC}^{21}$. Our non-presumptive analysis of global gene expression in young cTEC, aged cTEC, or aged cTEC in the regenerating thymus suggested that genes associated with cell size and shape dominated the dynamic landscape (Fig. 1). However, the size and shape of individual cTEC has been difficult to discern using conventional methods, thus obscuring any changes that might occur during age atrophy or regeneration.

Here, we used Confetti conditional labeling to accurately define the size and shape of individual TEC, as well as H2b:mCherry conditional labeling to unambiguously define TEC number and density without obstruction by other cell types. Our results reveal novel aspects of TEC biology (especially among cTEC) that broadly influence our interpretation of their normal function. The morphology of cTEC is absolutely unique, as far as we can tell, in all of metazoan biology. The closest cellular correlate may be Purkinje cells, but unlike these and other truly branched cells, which are characterized by serial bifurcations that eventually terminate, cTEC cell projections consist of labyrinthine, looping structures forming about 20-30 small cavities per cTEC, each of which contains about 2-10 lymphoid cells on average (e.g., about 150 lymphoid cells per cTEC). All cTEC from young thymus (other than those lining the capsule or blood vessels) are very similar in overall size and shape (defined in Fig. 2d and Supplementary Movie 2), represented by a slightly flattened ovoid with a long axis of approximately $75 \mu \mathrm{m}$, with the long axis generally perpendicular to the capsule. Based on this unimodal size, it appears that the overall dimensions of cTEC are specified genetically (within the constraints of the local microenvironment), much like any other cell type. In contrast, the organization of looping cell projections within cTEC is highly variable, and thus seems unlikely to be genetically specified. Instead, it appears these complex projections are defined and constrained by the shape of the lymphocytes that they contain. We believe that this shaping of cTEC projection morphology by lymphoid cells illustrates the well-recognized phenomenon of crosstalk ${ }^{7}$, where each cell type requires the presence of the other for normal development. The intimate wrapping of lymphocytes by cTEC projections, together with their unimodal size and shape, compels us to reflect on the potential relationship of cTEC to thymic nurse cells ${ }^{56}$, which are defined as a rare population of cTEC that completely enclose a variable number of immature lymphocytes. We believe that the apparent rarity may be explained by the very small proportion of cTEC expected to survive mechanical disruption of the tissue, based on the complex and fragile morphology we show here. While cTEC defined by Confetti do not completely engulf their associated lymphocytes, it is worth noting that this particular characteristic of nurse cells was demonstrated in vitro, not in $\operatorname{situ}^{56}$. Consequently, we now conclude that all cTEC essentially represent nurse cells or, stated another way, that nurse cells are no different than the average cTEC. In the context of cTEC acting as the matrix for lymphoid progenitor migration (reviewed in ref. ${ }^{4}$ ), we also note that the morphology of cTEC defined by Confetti suggests that lymphoid progenitors migrate through individual cTEC, rather than upon them, perhaps an esoteric distinction, but one that is consistent with the nurse cell concept of engulfment of lymphoid cells.

Defining the morphology of cTEC (and mTEC) allowed us to test the predictions of our aging/regeneration database (Fig. 1), which suggested that changes in cell projection morphology were dominant. Confetti labeling in 12-month-old mice showed that cortical atrophy is indeed characterized by contraction of cTEC cell projections, while regeneration correlates with their reextension. We note that neither contraction during aging nor regrowth during regeneration is uniform among all cTEC. In atrophy, cTEC contraction is most conspicuous in the outer regions of the cortex (Fig. 2g, i, and Supplementary Movies 1 and 2), resulting in a disproportionate increase in cTEC density in this area (Fig. 4). This same region corresponds to the site of intense lymphoid proliferative activity (Fig. 5, and refs. ${ }^{8,48}$ ), and thus, of intense anabolic metabolism. Notably, the autofluorescent inclusions that are hallmarks of metabolic damage during aging 37,38 are also concentrated here (Fig. $2 \mathrm{~h}$ and Supplementary Fig. 2e, f). We have previously shown that cTEC express low levels of the antioxidant enzyme catalase, and thus they rapidly accumulate oxidative damage ${ }^{57}$. Therefore, it makes sense that this process would be most conspicuous in the outer cortex, where intense anabolic metabolism occurs as the consequence of rapid lymphoblastic proliferation (Fig. 5, and refs. ${ }^{8,48}$ ). From all these observations, we conclude that cortical atrophy (and, therefore, thymic atrophy) results from a toxic cycle of anabolic lymphoid metabolism, cTEC susceptibility to the byproducts of that metabolism, accumulated metabolic damage in cTEC, and their resultant atrophy, a process that initiates in the sub-capsular cortex and progressively moves inward through the cortex with age.

Like degeneration with age, regeneration among cTEC after castration is also non-homogenous. Although the overall appearance of the cortex (as defined by Confetti) is dramatically improved (Fig. 2j), it is associated with significant hypertrophy in some cells, but little change in others. We are unable to predict whether heterogeneity among cTEC responses during regeneration reflects cells with differing levels of accumulated damage, or whether it is simply stochastic, although the former seems the more intuitive option. In any case, the regeneration that does occur is sufficient to fully reconstitute the cortex (and thus, the thymus), albeit transiently ${ }^{21}$. We believe that the transient nature of thymic regeneration ${ }^{21}$ is more consistent with cTEC growth, rather than proliferation, as a mechanism; cTEC generated de novo from stem/progenitor cells would be expected to have a 
a

Signaling by VEGF (R-MMU-194138,61/97 genes) mTOR signaling (R-MMU-165159 27/40 genes)

Interleukin-1 signaling (R-MMU-9020702, 53/93 genes) mTORC1-mediated signaling (R-MMU-166208,17/23 genes) MAPK6/MAPK4 signaling (R-MMU-5687128, 42/71 genes) Interleukin-1 family signaling (R-MMU-446652, 61/110 genes) Signaling by Hippo (R-MMU-2028269, 15/20 genes)

$\beta$-catenin indep. Wnt signaling (R-MMU-3858494, 64/118 genes) CLEC7A (Dectin-1) signaling (R-MMU-5607764, 47/84 genes) MET activates PTK2 signaling (R-MMU-8874081, 20/30 genes)
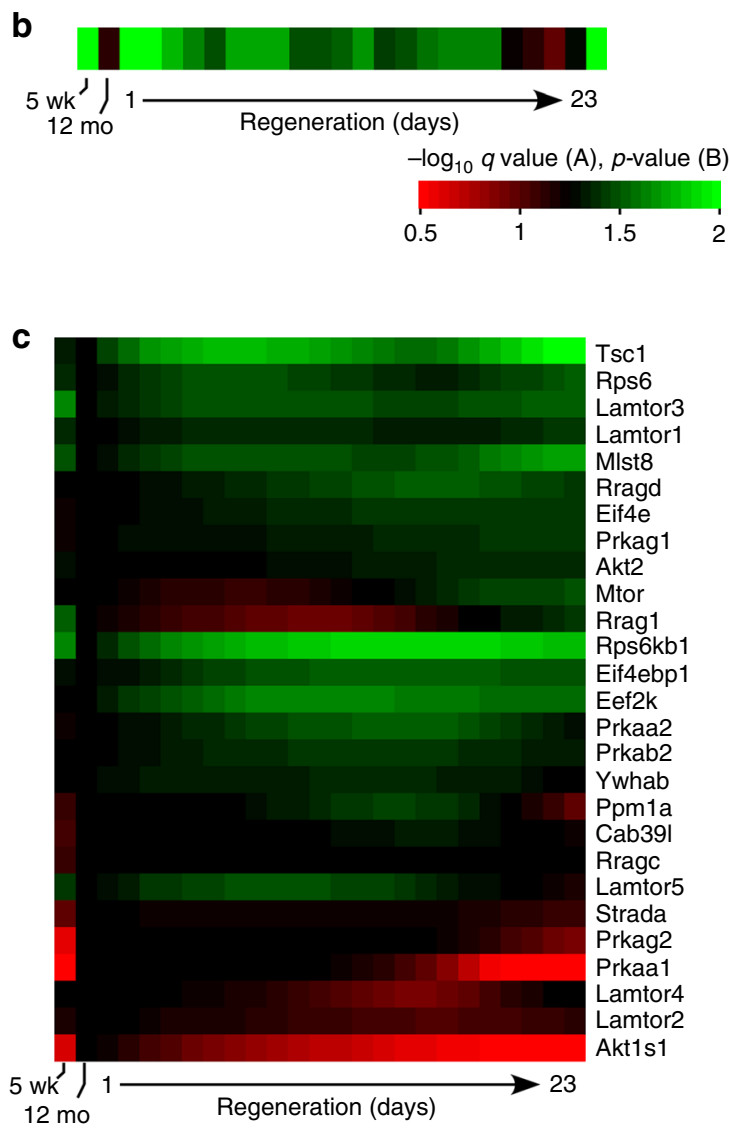

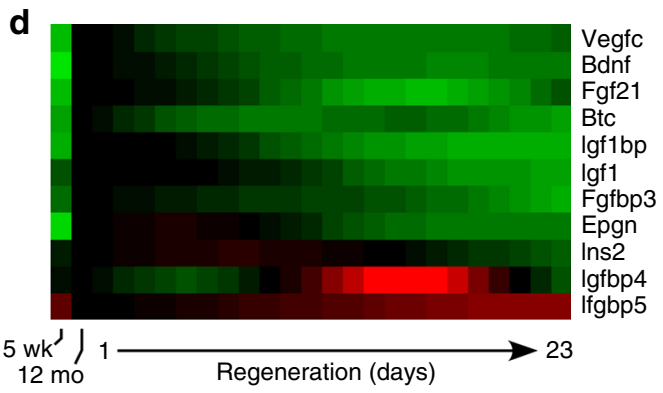

$\mathbf{e}$
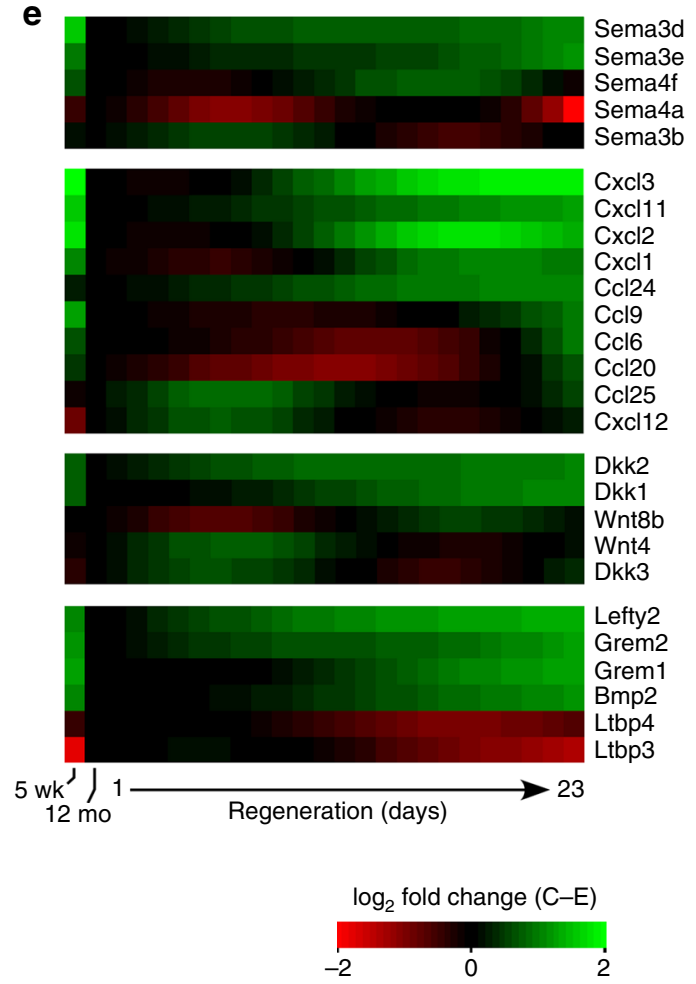

Fig. 6 Pathways regulating cell size or shape suggest control of cTEC morphology by medullary ligands. a-c Representation of data from cortical stroma, while d, e derive from medullary stroma. c-e Normalized to the 12-month-old untreated thymus, which also represents day 0 of the regeneration sequence. a The top 10 Reactome signaling pathways (sorted by hypergeometric $q$ value) mapping to genes expressed by cortical stroma. Reactome pathway designations, and the number of genes expressed in thymic stromal cells vs. the total genes in the pathway, are shown in parentheses. Note that 3 of the top 4 pathways impact mTor signaling, making mTor a prime candidate for changes in CTEC morphology during aging and regeneration. $\mathbf{b}$ Change in representation (determined by the Fisher exact test) of the mTor pathway in cortical stroma during atrophy and regeneration. $\mathbf{c}$ Dynamics of $\mathrm{mTor}$ pathway genes in cortical stroma during atrophy and regeneration. $\mathbf{d}$ Dynamic regulation of mTor-activating ligands by medullary stroma; particularly notable are Igf1 and Fgf21. e Medullary stroma also dynamically regulate other classical paracrine mediators of cell growth and morphology, including members of the Sema, chemokine, Wnt, and Tgfb signaling pathways. Source data are provided in Supplementary Data 1

relatively normal lifespan, and to degenerate much more slowly, instead of returning to the atrophied state within 2 weeks ${ }^{21}$. In contrast, changes in cell projection morphology can be easily accommodated by a 2 -week time frame. Our overall conclusion from analysis of dynamic gene expression (Fig. 1), cell morphology (Fig. 2), cell density (Fig. 4), and cell proliferation (Fig. 5) is that thymic atrophy and regeneration both reflect modulation of cTEC morphology (size), which occurs independently of appreciable levels of cell death or proliferation. We wish to point out that we refer here only to the accelerated atrophy of the thymus seen at a relatively young age (for instance, the $75 \%$ reduction in cellularity seen by 12 months of age in mice, and proportionally in all other species with a thymus). It is possible, if not likely, that senescence-associated cell death plays an increasing role at much later age, as it does in other tissues.
In contrast to the relatively unimodal gross size and shape found in young cTEC, mTEC display a wide diversity of sizes shapes that do not cluster into identifiable groups, but instead represent a broad, continuous spectrum (Fig. 3). It is unclear whether or not extremes in this distribution are functionally or genetically distinct. However, it is interesting to contemplate that in the cortex, where cTEC are all essentially identical, lymphoid progenitor cells follow a synchronous path involving proliferation, commitment to the $\mathrm{T}$ lineage, TCR/MHC screening, etc. In contrast, in the medulla, where mTEC morphology varies widely, multiple $\mathrm{T}$ lineages with distinct functional capacities diverge (helper, cytotoxic, regulatory, etc). Thus, it seems worth considering that mTEC heterogeneity may reflect distinct roles in specifying different $\mathrm{T}$ cell functions, a hypothesis that is readily testable using Confetti-labeled mTEC and various $\mathrm{T}$ lineage markers. 
In addition to visualizing the distinct morphologies of individual TEC, our studies also reveal a previously unrecognized role for medullary signals in the maintenance and regulation of cTEC morphology. While classical response pathways determining cell size and shape (most notably, Mtor) are dynamically regulated by cTEC themselves during atrophy and regeneration (Fig. 6), the inductive ligands are generally not produced in that compartment, either by stromal or lymphoid cells. This class of ligands can be of endocrine origin, but it is important to note that the thymic cortex is immunologically privileged, protected by a blood vessel barrier that tightly restricts entry of exogenous substances $^{53}$. The medulla, in contrast, is quite permeant to exogenous molecules ${ }^{53}$, and thus is much more likely to be exposed to endocrine or other systemic cues. We find that a number of ligands relevant to pathways that change in CTEC during atrophy and regeneration are produced by medullary stroma, and are dynamically regulated during aging and regeneration (Fig. 6). This implies the existence of an endocrine/paracrine signaling axis, were the medulla responds to peripheral (endocrine) cues by signaling to the cortex in paracrine fashion, at least one consequence of which is modulation of cTEC morphology. Dependence of the cortex on the presence of a medulla was first noted more than 50 years ago ${ }^{58}$, although the nature of this dependence has still not been elucidated. Our data suggests that it may be explained, at least in part, by dependence of cTEC on morphogenic signals derived from medullary stroma, either in the steady state or during atrophy/regeneration. How exogenous substances entering the thymus via the medulla are subsequently prevented from entering the cortex ${ }^{53}$, while intrinsic medullary products (for instance, the chemokines CCL21 and CCL2 $5^{59}$ ) can readily do so is completely unclear. In this respect, it is interesting to note that a formal barrier between medulla and cortex has never been defined, even though simple histological analysis indicates that it must exist, since the lymphoid and stromal contents of these compartments do not mix. Regardless of how this barrier is formed, our findings suggest that the cortical response seen after castration does not result directly from androgen signaling to cTEC, but rather reflects responses by medullary stroma to the endocrine environment (possibly including androgen signaling), subsequently resulting in regulation of cTEC morphology. Our data also suggest that even though mTEC morphology and density (Figs. 3 and 4 ) and the size of the medullary compartment $^{21}$ do not change much during aging, the ability of medullary stroma to produce signals controlling cTEC morphology does diminish with age, in a manner that is transiently restored ${ }^{21}$ by the systemic response to castration.

\section{Methods}

Mice. C57Bl/6 mice (JAX strain 000664), Foxn1[Cre] knockin mice (JAX strain $018448^{35}$ ), Rosa[Confetti] mice (JAX strain $013731^{34}$ ), or Rosa[H2b:mCherry] mice (JAX strain $023139^{39}$ ) were purchased from Jackson Laboratories, and bred or crossed at Scripps. All experimental mice were male. For surgical castration, animals were anesthetized with ketamine $(120 \mathrm{mg} / \mathrm{kg})$ and xylazine $(16 \mathrm{mg} / \mathrm{kg})$ administered intraperitoneally. The surgical site was sterilized by swabbing with Betadine and then ethanol, and a 3-4 mm incision was made through the skin and tunica surrounding testicle using a scalpel. The testes were exposed and all associated vessels were clamped with a hemostat, followed by ligation using 4-0 silk sutures, and excision of the testes. The site was then closed using Vetbond adhesive ( $3 \mathrm{M}$, St. Paul, MN, USA). The procedure was repeated individually for each testicle. Animals were given $0.2 \mathrm{ml}$ of sterile saline subcutaneously, placed in a sternal recumbent position on a heating pad, and monitored until they became ambulatory. Buprenorphine $(0.05 \mathrm{mg} / \mathrm{kg})$ was administered to relieve pain immediately after the mice became ambulatory, and every $8 \mathrm{~h}$ thereafter until activity had returned to normal. All animal use was compliant with ethical standards, and was reviewed and approved by the Scripps Institutional Animal Care and Use Committee.

Cell, tissue, and RNA isolation. These procedures were performed using our previously validated methods ${ }^{21,60}$. Microdissected tissue and lymphoid cells were paired, in that tissue was isolated from one lobe of the thymus, and lymphoid cells from the other. For tissue isolation, intact thymus lobes were removed and immediately placed in ice cold OCT mounting medium, then frozen. Transverse $20 \mu \mathrm{m}$ sections were cut from the middle third of the organ (A/P axis) and mounted on PEN membrane slides (Leica). Sections selected for microdissection were chosen to have cortical regions $400-600 \mu \mathrm{m}$ deep, medullary regions at least $200 \mu \mathrm{m}$ deep, and symmetrical cortical/medullary borders. Tissue was fixed in cold acetone/ethanol $(3: 1 \mathrm{v} / \mathrm{v})$, rehydrated through graded ethanol, stained with cresyl violet (LCM Staining Kit, Ambion), and dehydrated through graded ethanol. Slides were dried at room temperature and immediately used for microdissection on a Leica AS LMD system. Sections adjacent to those used for microdissection were mounted on glass slides for archival purposes. Additional sections $100 \mu \mathrm{m}$ above and below microdissected sections were also mounted on glass slides and examined to rule out tissue irregularities outside microdissected plane. Cortical subregions were prepared from tissues as described above, with each subregion representing $15 \%$ of the total cortical depth. RNA was prepared using RNAqueous Micro kits (Ambion). Each independent RNA pool included tissue from multiple sections and mice.

For lymphoid cells, thymus lobes were removed and placed immediately in ice cold medium (Hanks' balanced salt solution, 5\% FBS, $10 \mu \mathrm{g} / \mathrm{ml}$ DNAse), with all subsequent steps at $4^{\circ} \mathrm{C}$. Single cell suspensions were stained with labeled antibodies (below), followed by cell sorting. Sort gates for medullary lymphoid cells were $\mathrm{CD}^{\text {hi }}$ AND $\mathrm{CD}^{4} 5^{+}$, and for cortical lymphoid cells were $\mathrm{CD} 3^{-/ \mathrm{lo}}$ AND $\mathrm{CD}^{+} 5^{+} \mathrm{AND}\left(\mathrm{CD} 90^{+} \mathrm{OR} \mathrm{CD} 117^{+}\right)$. Biotinylated CD3 antibody was from eBioscience, catalog \# 13-0031-86, lot \# E 0234801631 used at 1:50 dilution, followed by Alexa 647-streptavidin from Molecular Probes, catalog number S11229 , lot \# 65A1-1, used at $2 \mu \mathrm{g} / \mathrm{ml}$. PE-conjugated CD45 antibody was from Caltag, catalog \# RM6404, lot \# 01020803, used at 1:100 dilution. Alexa-647conjugated CD90 antibody was from BioLegend, catalog \# 105318, lot \# B175617, used at 1:25 dilution. FITC-conjugated CD117 was from eBioscience, catalog \# 11-1171, lot \# E00475-330, used at 1:200 dilution. Dead cells were excluded using DAPI. Cells for microarray analysis were selected to be $>99 \%$ pure, and were generally $>99.8 \%$ pure. RNA was prepared using RNeasy Mini Kits (Qiagen) according to recommended protocols.

In vitro transcription, RNA labeling, and array hybridization. These were carried out by the Genomics Core Facility at The Scripps-Florida Research Institute Each RNA template consisted of at least three pooled RNA samples of the same type (e.g., cortical lymphocytes) but from different thymuses, in order to minimize sample variation. $0.2-1.0 \mu \mathrm{g}$ of total RNA was used for a single cycle of in vitro transcription. Biotin-labeled cRNA probes were then prepared as recommended by the manufacturer (Affymetrix) using oligo-dT primers, SuperScript, and BioArray High Yield RNA Transcript kits (Affymetrix). Fragmentation/hybridization to MOE430v2.0 arrays (Affymetrix) were performed according to standard Affymetrix protocols. Imaging was performed on a Hewlett Packard GeneArray Scanner MIAME-compliant raw data has been deposited into the NCBI Gene Expression Omnibus database (GSE132136). The total raw data includes 156 arrays. Each data point includes 4 microarrays, representing lymphoid cells or microdissected tissue from cortex or medulla. Twenty serial time points are included, including 5-weekold mice, untreated 12-month-old mice (day 0 of regeneration), or castrated mice at each day from day 6 to day 23 after surgery. At least one array was generated for each sample type (cortical or medullary, lymphoid cells or tissue) on each day, with additional replicates at key inflection points: young ( 5 independent arrays per cell/ tissue type), day 0 ( 4 independent arrays per cell type), day 6 ( 3 independent arrays per cell type), day 7 ( 2 independent arrays per cell type), day 12 (3 independent arrays per cell type), day 16 ( 2 independent arrays per cell type), day 17 ( 3 independent arrays per cell type), and day 20 independent arrays per cell type).

Identification of genes expressed by stromal cells. All processing was per-

formed in $\mathrm{R}^{61}$. All 156 .cel files were normalized using the rma() function from the $\mathrm{R}$ package affy ${ }^{62}$, with the normalize option set to TRUE. A permissive (minimum of false negatives) list of genes designated as being expressed in the thymus

(stromal or lymphoid) was first defined as those genes in tissue microarrays that received a majority of non-absent detection calls (i.e., Present or Marginal, using the mas 5 calls() function in the R package affy) at any time point where 3 or more independent replicates were present, or, where the signal value was above the chip median in the majority at any time point where 3 or more independent replicates were present. For each probeset in this list, 3rd degree polynomial splines were fit to the regeneration time-course for each sample type (days 0 to 23 post-castration) using the ns ( $\mathrm{R}$ base package splines), rlm (R package MASS ${ }^{63}$ ), and predict (base package stats) functions, with the hampel method for outlier detection and down weighting. Internal knots were placed at days 6 and 17. This splined (smoothed) data for all expressed genes (tissue or lymphoid) was used for subsequent processing. Stromal gene expression was calculated using our previously published approach ${ }^{60}$, using the formula stromal expression = [tissue signal-(lymphoid signal $\times$ lymphoid proportion $)] /(1$-lymphoid proportion), where tissue signal is the measured (splined) tissue signal values, lymphoid signal is the measured (splined) lymphoid signal values, and lymphoid proportion is the average ratio of lymphoid to tissue signal values for a manually curated list of known lymphoid-specific genes $^{60}$. The resulting calculated stromal values were then quantile renormalized to 
the corresponding spline fit data for each day. When data from young thymuses were included in the analysis, stromal signals were calculated similarly but using measured tissue and lymphoid values in place of spline-fit values. For analyses where a minimum of false positive stromal genes was desirable (for instance, Fig. 1), at the expense of more false negatives, a high confidence stromal gene list was defined by setting a minimum tissue:lymphoid ratio threshold equal to the mean +2.5 s.d. for the lymphoid-specific list of genes. For analyses where a minimum of false negatives was desirable (for instance, Fig. 6), at the expense of more false positives, this threshold was set to mean +2.0 s.d. Additional details specific to Figs. 1 or 6, but not both, are provided in the corresponding Results sections.

Enrichment analysis. Gene Ontology enrichments were performed using the hyperGTest() function from the R package GOstats ${ }^{64}$ with the conditional option set to TRUE, and with ontology mappings from the org.Mm.eg.db package ${ }^{65}$. Reactome enrichments were performed using the enrichPathway function from the ReactomePA package in $\mathrm{R}^{66}$, with all genes as background. Overrepresentation was calculated using the fisherexact function in the base R package stats.

Isolation and preparation of tissue for microscopic imaging. After euthanasia, the thymus was removed and placed immediately into ice-cold $2-4 \%$ formaldehyde (Polysciences \#18814) in PBS, followed by gentle rotation at $4{ }^{\circ} \mathrm{C}$ for $6-16 \mathrm{~h}$. Graded sucrose infiltration (10/20/30\% in PBS) was then performed for $\sim 24 \mathrm{~h}$ at $4{ }^{\circ} \mathrm{C}$, followed by cryogenic mounting in OCT. Tissues were sectioned to the appropriate thickness (depending on the application, $5 \mu \mathrm{m}$ to $150 \mu \mathrm{m}$ ) and mounted on positively charged glass microscope slides. Tissues were never allowed to dry, even momentarily. OCT was removed by incubation at room temperature for $1 \mathrm{~h}$ in PBS, followed by washing in PBS, mounting, and coverslipping. For tissue $>10 \mu \mathrm{m}$ thick, mylar spacers of an appropriate thickness were used between the slide and coverslip to prevent compression of the tissue.

Confocal microscopy. Imaging was performed using a Leica SP5 Resonant Scanner (housed at the Max Planck Florida Institute, Jupiter, FL) equipped with $405 \mathrm{~nm}, 458 \mathrm{~nm}, 561 \mathrm{~nm}$, and $633 \mathrm{~nm}$ diode lasers, as well as an argon ion laser producing several spectral lines (most relevant, $488 \mathrm{~nm}$ and $514 \mathrm{~nm}$ ). For virtually all studies shown here, a $20 \times$ glycerol, long working distance immersion objective was used, and 8-bit gray scale images were obtained.

Confetti image acquisition. The Confetti allele encodes three fluorescent proteins (membrane Cfp, cytoplasmic Yfp, and cytoplasmic Rfp) that are useful for defining the full extent of labeled cells, and a fourth (nuclear Gfp) that was of limited value to the present studies. To minimize the detection of nGfp while acquiring the other Confetti colors, and simultaneously including a channel for subtraction of autofluorescent age pigments, a three stage sequential scan was used. All three scans used identical collection windows of 463-509 nm (window 1), 519-556 nm (window 2), and 566-628 nm (window 3). In the first scan, $405 \mathrm{~nm}$ and $458 \mathrm{~nm}$ lasers were used simultaneously (since neither of them was optimal) to excite both mCfp and autofluorescent pigments, corresponding to collection windows 1 and 3 , respectively. Note that autofluorescent pigments of age excite maximally in the UV/ violet range but emit across a broad spectrum of wavelengths, and thus, window 3 contained exclusively autofluorescent activity. In the second scan, a $514 \mathrm{~nm}$ laser was used to maximally excite cYfp (and minimally, nGfp), which was collected in window 2 (again, biased in favor of cYfp). In the third scan, a $561 \mathrm{~nm}$ laser was used to excite cRfp, collected in window 3. For full color displays, mCfp is presented as blue, cYfp as green, and cRfp as red. When an additional immunofluorecent stain was added, the fluochrome was Alexa 633 or Alexa 647 excited by the $633 \mathrm{~nm}$ laser, and a fourth detection window of $638 \mathrm{~nm}-700 \mathrm{~nm}$ was collected simultaneously with cYfp in the second stage scan, since Yfp and these far red dyes were spectrally non-overlapping.

Confetti cTEC image analysis. All image analysis in this study was performed using ImageJ software ${ }^{67,68}$ with associated plugins, as noted. Morphological analysis of cTEC was a particular challenge, due to their large size (and thus, frequent truncation by the volume boundaries), the small diameter of their extensive cell projections, and extensive degeneration with age. The following is a general description, although some small adjustments may have been applied non-identically, as necessary, as described below. Individual cells (defined by any single color surrounded by cells of other colors) contained wholly within the image volume were identified, and a corresponding smaller volume containing the entire cell was cropped out. Contrast enhancement (Process $>$ Enhance Contrast) was applied ( $0-3 \%$ saturation, as appropriate for any given cell), and a 3D median filter (Process $>$ Filters $>$ Median 3D, $x . y$ radii $=2, z$ radius $=1$ ) was applied. Segmentation was performed (Plugins $>$ boneJ $>$ Optimize Threshold ${ }^{69}$ to generate a binary image, and surrounding cells or parts of cells were removed (Plugins $>$ boneJ $>$ Purify). In some cases, additional noise removal (Process $>$ Noise $>$ Remove Outliers, or Process $>$ Noise $>$ Despeckle) or background subtraction (Process $>$ Subtract Background) may have been applied to generate a clean image for quantitation. Cell parameters, including volume, surface area, feret diameter, etc, were then calculated (Plugins $>3 \mathrm{D}>3 \mathrm{D}$ Manager $>$ Measure 3D) using 3D manager ${ }^{70}$.
Confetti mTEC image analysis. The first step in this process was to use tissue from (young) mice carrying one Rosa26 Confetti allele and one Rosa26 H2b: mCherry allele (as well as Foxn 1[Cre]) to validate the conditions by which individual mTEC could be distinguished within the tightly packed medullary compartment. The approach was refined by manual evaluation of $\mathrm{H} 2 \mathrm{~b}$ :mCherry overlaid onto the results, until it was clear (using a subset of images) that the approach was generally identifying objects that contained one, and only one, nucleus. For this analysis, only the cYfp channel was used to identify mTEC, because cRfp was in the same image channel as mCherry (separable by morphology), and mCfp resulted in a hollow image that was not readily amenable to counting. The following defines the process, which varied little to none between images. Process $>$ Enhance Contrast (1\% saturation), background subtraction (Process $>$ Subtract Background, rolling ball radius $=10$, sliding paraboloid), and median 3D filtering (Process $>$ Filters $>$ Median $3 \mathrm{D}, x, y$ radii $=2, z$ radius $=1$ ) were applied to further enhance the Yfp image and distinguish it from background or noise. A copy of this file was made for watershedding, as described later. 3D minimum filtering (Process $>$ Filters $>$ Minimum 3D, $x, y$ radii $=2, z$ radius $=1$ ) was applied to facilitate separation into individual objects. Next, the Plugins $>3 \mathrm{D}>$ 3D Edge and Symmetry filter was applied (alpha Canney $=0.3$, radius $=10$, normalization $=10$, scaling $=2$, with improved seed detection) to define individual objects (cells). The resulting symmetry smoothed file was restored to 8-bit pixel depth, and brightness levels were enhanced $(\min =5, \max =45)$. Plugins $>3 \mathrm{D}>3 \mathrm{~d}$ Maximum Finder $(x, y$ radii $=25, z$ radius $=10$, noise $=75)$ was then used to assign a single peak to each object, for use as a seed for watershedding. The image copy described above was smoothed (Plugins $>$ Process $>$ Smooth 3D, Gaussian value $=$ 0.5 ), and the watershed was applied to this image with the peaks as seeds, seeds threshold $=50$, and image threshold $=20$. Analyze $>3 \mathrm{D}>3 \mathrm{D}$ Objects Counter (threshold and size minima $=1$, exclude on edges, with objects boxes) was applied and the resulting image was restored to pixel density and size. 3D parameters were generated using Plugins $>3 \mathrm{D}>3 \mathrm{D}$ Manager $>$ Measure $3 \mathrm{D}$ with default parameters. These measurements, together with $\mathrm{H} 2 \mathrm{~b}$ :mCherry co-expression (above), were used to define filters to remove objects (cells) containing no nucleus or more than one nucleus. For the analysis presented in Fig. 3, object volume was constrained such that the lower threshold was greater than the average volume of an mTEC nucleus plus $3 \sigma$ (i.e., $>532 \mu \mathrm{m}^{3}$ ), while the upper threshold was set to exclude objects in the top $5 \%$ of three manually selected parameters (feret diameter, compactness, and volume:ellipsoid ratio). For Fig. 3, kernel density estimates were generated using the kde() function from the R package $\mathrm{ks}^{71}$.

Image analysis for measurement of TEC density. Raw images generally consisted of $0.25 \times 0.25 \times 0.1 \mathrm{~mm}$ confocal image stacks. In order to facilitate automated counting, image stacks were processed using a 3D median filter (radii $=2$ ), rolling ball radius background subtraction (radius $=25$ ), and noise reduction (Process $>$ Noise $>$ Remove outliers, with radius $=5$ and threshold $=1$ ). The processed stack was then thresholded (Process $>$ Adjust $>$ Threshold, minimum $=10$, maximum $=255$ ), the resulting stack was inverted, and then subtracted from the original image. Noise reduction (above) was then carried out once more to remove any small objects (noise) prior to application of the $3 \mathrm{D}$ maximum finder ${ }^{70}$ with $x y$ radius $=10, z$ radius $=3$, noise $=10$, to define a point representing the fluorescence maximum of each nucleus. Accuracy was manually evaluated for each stack by overlaying a colorized version of the fluorescence maximum point onto the original H2b:mCherry image. Final quantitation represents multiple distinct tissue volumes from at least 3 independent thymuses in each anatomic compartment at each age. Note that capsule proximal volumes were excluded from cortical counting, due to the $3 \mathrm{D}$ curvature of the organ.

In vivo metabolic labeling of DNA. One hour prior to euthanasia, mice received a single intraperitoneal injection of 5-ethnyl-2'-deoxyuridine (ThermoFisher A10044) at a dose equimolar to that previously described ${ }^{8}$ for $\mathrm{BrdU}(40 \mu \mathrm{g} / \mathrm{g}$ body weight). Tissue was then fixed in formaldehyde and processed in graded sucrose for cryosectioning, as described above. Tissue sections (50 $\mu \mathrm{m}$ thick) were permeabilized for $1 \mathrm{~h}$ at room temperature in PBS containing 3\% BSA and 0.5\% Tween-20, followed by copper catalyzed click labeling with an Alexa 488 azide conjugate (ThermoFisher \#C10337) for $45^{\prime}$ at room temperature, several washes in PBS/BSA, and confocal imaging.

Reporting summary. Further information on research design is available in the Nature Research Reporting Summary linked to this article.

\section{Data availability}

The datasets generated during and analyzed during the current study are hosted by GEO GSE132136. The source data underlying Figs. $2 \mathrm{k}-\mathrm{m}, 3 \mathrm{~d}, 4 \mathrm{e}-\mathrm{f}$, and $5 \mathrm{~g}, \mathrm{k}, \mathrm{l}$, and $\mathrm{m}$ are provided in the Source Data file.

Received: 1 April 2019 Accepted: 7 August 2019

Published online: 27 September 2019 


\section{References}

1. Foss, D. L., Donskoy, E. \& Goldschneider, I. The importation of hematogenous precursors by the thymus is a gated phenomenon in normal adult mice. J. Exp. Med. 193, 365-374 (2001)

2. Takahama, Y., Ohigashi, I., Baik, S. \& Anderson, G. Generation of diversity in thymic epithelial cells. Nat. Rev. Immunol. 17, 295-305 (2017).

3. Abramson, J. \& Anderson, G. Thymic epithelial cells. Annu. Rev. Immunol. 35, 85-118 (2017).

4. Petrie, H. T. \& Zúñiga-Pflücker, J. C. Zoned out: functional mapping of stromal signaling microenvironments in the thymus. Annu. Rev. Immunol. 25, 649-679 (2007).

5. Klug, D. B. et al. Interdependence of cortical thymic epithelial cell differentiation and T-lineage commitment. Proc. Natl Acad. Sci. USA 95, 11822-11827 (1998)

6. Desanti, G. E. et al. Developmentally regulated availability of RANKL and CD40 ligand reveals distinct mechanisms of fetal and adult cross-talk in the thymus medulla. J. Immunol. 189, 5519-5526 (2012).

7. van Ewijk, W., Shores, E. W. \& Singer, A. Crosstalk in the mouse thymus. Immunol. Today 15, 214-217 (1994).

8. Lind, E. F., Prockop, S. E., Porritt, H. E. \& Petrie, H. T. Mapping precursor movement through the postnatal thymus reveals specific microenvironments supporting defined stages of early lymphoid development. J. Exp. Med. 194, 127-134 (2001).

9. Prockop, S. E. et al. Stromal cells provide the matrix for migration of early lymphoid progenitors through the thymic cortex. J. Immunol. 169, 4354-4361 (2002).

10. Prockop, S. E. \& Petrie, H. T. Regulation of thymus size by competition for stromal niches among early T cell progenitors. J. Immunol. 173, 1604-1611 (2004).

11. Kyewski, B. \& Klein, L. A central role for central tolerance. Annu. Rev. Immunol. 24, 571-606 (2006).

12. Gray, H., Williams, P. L. \& Bannister, L. H. Gray's anatomy: the anatomical basis of medicine and surgery. (Churchill Livingstone, New York, 1995).

13. Clark, S. L. The thymus in mice of strain 129/J, studied with the electron microscope. Am. J. Anat. 112, 1-33 (1963).

14. Hoshino, T. Electron microscopic studies of the epithelial reticular cells of the mouse thymus. Z. Zellforsch. Mikros. Anat. 59, 513-529 (1963).

15. van Ewijk, W. Cell surface topography of thymic microenvironments. Lab. Invest. 59, 579-590 (1988).

16. van Ewijk, W. et al. Thymic microenvironments, 3-D versus 2-D. Semin Immunol. 11, 57-64 (1999).

17. Farr, A. G. \& Braddy, S. C. Patterns of keratin expression in the murine thymus. Anat. Rec. 224, 374-378 (1989).

18. Steinmann, G. G., Klaus, B. \& Müller-Hermelink, H. K. The involution of the ageing human thymic epithelium is independent of puberty. A morphometric study. Scand. J. Immunol. 22, 563-575 (1985).

19. Domínguez-Gerpe, L. \& Rey-Méndez, M. Age-related changes in primary and secondary immune organs of the mouse. Immunol. Invest 27, 153-165 (1998).

20. Berent-Maoz, B., Montecino-Rodriguez, E. \& Dorshkind, K. Genetic regulation of thymocyte progenitor aging. Semin. Immunol. 24, 303-308 (2012).

21. Griffith, A. V., Fallahi, M., Venables, T. \& Petrie, H. T. Persistent degenerative changes in thymic organ function revealed by an inducible model of organ regrowth. Aging Cell 11, 169-177 (2012).

22. Haynes, B. F., Markert, M. L., Sempowski, G. D., Patel, D. D. \& Hale, L. P. The role of the thymus in immune reconstitution in aging, bone marrow transplantation, and HIV-1 infection. Annu. Rev. Immunol. 18, 529-560 (2000).

23. Haynes, B. F., Sempowski, G. D., Wells, A. F. \& Hale, L. P. The human thymus during aging. Immunol. Res. 22, 253-261 (2000).

24. Nikolich-Žugich, J. Aging of the T cell compartment in mice and humans: from no naive expectations to foggy memories. J. Immunol. 193, 2622-2629 (2014).

25. Henderson, J. On the relationship of the thymus to the sexual organs: I. The influence of castration on the thymus. J. Physiol. 31, 222-229 (1904).

26. Utsuyama, M. \& Hirokawa, K. Hypertrophy of the thymus and restoration of immune functions in mice and rats by gonadectomy. Mech. Ageing Dev. 47, 175-185 (1989).

27. Sutherland, J. S. et al. Activation of thymic regeneration in mice and humans following androgen blockade. J. Immunol. 175, 2741-2753 (2005).

28. Rode, I. \& Boehm, T. Regenerative capacity of adult cortical thymic epithelial cells. Proc. Natl Acad. Sci. USA 109, 3463-3468 (2012).

29. Klug, D. B. et al. Transgenic expression of cyclin D1 in thymic epithelial precursors promotes epithelial and T cell development. J. Immunol. 164, 1881-1888 (2000).

30. Henry, L. \& Anderson, G. Epithelial-cell architecture during involution of the human thymus. J. Pathol. 152, 149-155 (1987).

31. Plećas-Solarović, B., Pesić, V., Radojević, K. \& Leposavić, G. Morphometrical characteristics of age-associated changes in the thymus of old male Wistar rats. Anat. Histol. Embryol. 35, 380-386 (2006).
32. Brelinska, R., Malendowicz, L. K., Malinska, A. \& Kowalska, K. Characteristics of age-related changes in rat thymus: morphometric analysis and epithelial cell network in various thymic compartments. Biogerontology 9, 93-108 (2008).

33. Ashburner, M. et al. Gene ontology: tool for the unification of biology. The Gene Ontology Consortium. Nat. Genet. 25, 25-29 (2000).

34. Snippert, H. J. et al. Intestinal crypt homeostasis results from neutral competition between symmetrically dividing Lgr5 stem cells. Cell 143, 134-144 (2010).

35. Gordon, J. et al. Specific expression of lacZ and cre recombinase in fetal thymic epithelial cells by multiplex gene targeting at the Foxn1 locus. $B M C$ Dev. Biol. 7, 69 (2007).

36. Seehafer, S. S. \& Pearce, D. A. You say lipofuscin, we say ceroid: defining autofluorescent storage material. Neurobiol. Aging 27, 576-588 (2006).

37. Terman, A. \& Brunk, U. T. Lipofuscin: mechanisms of formation and increase with age. APMIS 106, 265-276 (1998).

38. Porta, E. A. Pigments in aging: an overview. Ann. NY Acad. Sci. 959, 57-65 (2002).

39. Peron, S. P., Freeman, J., Iyer, V., Guo, C. \& Svoboda, K. A cellular resolution map of barrel cortex activity during tactile behavior. Neuron 86, 783-799 (2015).

40. Sakata, M., Ohigashi, I. \& Takahama, Y. Cellularity of thymic epithelial cells in the postnatal mouse. J. Immunol. 200, 1382-1388 (2018).

41. Scheiff, J. M., Cordier, A. C. \& Haumont, S. Epithelial cell proliferation in thymic hyperplasia induced by triiodothyronine. Clin. Exp. Immunol. 27, 516-521 (1977).

42. Gillard, G. O. \& Farr, A. G. Features of medullary thymic epithelium implicate postnatal development in maintaining epithelial heterogeneity and tissuerestricted antigen expression. J. Immunol. 176, 5815-5824 (2006).

43. Gray, D., Abramson, J., Benoist, C. \& Mathis, D. Proliferative arrest and rapid turnover of thymic epithelial cells expressing Aire. J. Exp. Med. 204, 2521-2528 (2007)

44. Michel, C. et al. Revisiting the road map of medullary thymic epithelial cell differentiation. J. Immunol. 199, 3488-3503 (2017).

45. Gray, D. H. et al. Developmental kinetics, turnover, and stimulatory capacity of thymic epithelial cells. Blood 108, 3777-3785 (2006).

46. Dudakov, J. A. et al. Interleukin-22 drives endogenous thymic regeneration in mice. Science 336, 91-95 (2012).

47. Salic, A. \& Mitchison, T. J. A chemical method for fast and sensitive detection of DNA synthesis in vivo. Proc. Natl Acad. Sci. USA 105, 2415-2420 (2008)

48. Penit, C. Localization and phenotype of cycling and post-cycling murine thymocytes studied by simultaneous detection of bromodeoxyuridine and surface antigens. J. Histochem. Cytochem. 36, 473-478 (1988).

49. Laplante, M. \& Sabatini, D. M. mTOR signaling in growth control and disease. Cell 149, 274-293 (2012).

50. Gonzalez, S. \& Rallis, C. The TOR signaling pathway in spatial and temporal control of cell size and growth. Front. Cell Dev. Biol. 5, 61 (2017).

51. Kennedy, B. K. \& Lamming, D. W. The mechanistic target of rapamycin: the grand conducTOR of metabolism and aging. Cell Metab. 23, 990-1003 (2016).

52. Goldberg, E. L. et al. Lifespan-extending caloric restriction or mTOR inhibition impair adaptive immunity of old mice by distinct mechanisms. Aging Cell 14, 130-138 (2015)

53. Raviola, E. \& Karnovsky, M. J. Evidence for a blood-thymus barrier using electron-opaque tracers. J. Exp. Med. 136, 466-498 (1972).

54. Chu, Y. W. et al. Exogenous insulin-like growth factor 1 enhances thymopoiesis predominantly through thymic epithelial cell expansion. Blood 112, 2836-2846 (2008).

55. Youm, Y. H., Horvath, T. L., Mangelsdorf, D. J., Kliewer, S. A. \& Dixit, V. D. Prolongevity hormone FGF21 protects against immune senescence by delaying age-related thymic involution. Proc. Natl Acad. Sci. USA 113 1026-1031 (2016)

56. Wekerle, H., Ketelsen, U. P. \& Ernst, M. Thymic nurse cells. Lymphoepithelial cell complexes in murine thymuses: morphological and serological characterization. J. Exp. Med. 151, 925-944 (1980).

57. Griffith, A. V. et al. Metabolic damage and premature thymus aging caused by stromal catalase deficiency. Cell Rep. 12, 1071-1079 (2015).

58. Metcalf, D. in The Thymus (eds. Defendi, V. \& Metcalf, D.) 53-74 (The Wistar Institute Press, Philadelphia, 1964).

59. Kwan, J. \& Killeen, N. CCR7 directs the migration of thymocytes into the thymic medulla. J. Immunol. 172, 3999-4007 (2004).

60. Griffith, A. V. et al. Spatial mapping of thymic stromal microenvironments reveals unique features influencing $\mathrm{T}$ lymphoid differentiation. Immunity 31, 999-1009 (2009).

61. R Development Core Team. R: A language and environment for statistical computing. R Foundation for Statistical Computing (2017)

62. Gautier, L., Cope, L., Bolstad, B. M. \& Irizarry, R. A. affy-analysis of Affymetrix GeneChip data at the probe level. Bioinformatics 20, 307-315 (2004). 
63. Venables, W. N. \& Ripley, B. D. Modern applied statistics with S. (Springer, New York, 2002)

64. Falcon, S. \& Gentleman, R. Using GOstats to test gene lists for GO term association. Bioinformatics 23, 257-258 (2007).

65. Carlson, M. org.Mm.eg.db: Genome wide annotation for Mouse (R package version 3.4.1) (2017).

66. Yu, G. \& He, Q. Y. ReactomePA: an R/Bioconductor package for reactome pathway analysis and visualization. Mol. Biosyst. 12, 477-479 (2016).

67. Schneider, C. A., Rasband, W. S. \& Eliceiri, K. W. NIH Image to ImageJ: 25 years of image analysis. Nat. Methods 9, 671-675 (2012).

68. Schindelin, J. et al. Fiji: an open-source platform for biological-image analysis. Nat. Methods 9, 676-682 (2012).

69. Doube, M. et al. BoneJ: free and extensible bone image analysis in ImageJ. Bone 47, 1076-1079 (2010).

70. Ollion, J., Cochennec, J., Loll, F., Escudé, C. \& Boudier, T. TANGO: a generic tool for high-throughput 3D image analysis for studying nuclear organization. Bioinformatics 29, 1840-1841 (2013).

71. Duong, T. ks: Kernel density estimatoin and kernel discriminant analysis for multivariate data in R. J. Stat. Softw. 21, 1-16 (2007).

\section{Acknowledgements}

We sincerely thank Drs. Dawen Cai, Andrew Farr, Brock Grill, Rafael Irizarry, Joseph Kissil, Douglas Roossien, Casey Weaver, and Long Yan for helpful discussions. This work was supported by PHS grant AI130303 to HTP, and by funds from the Scripps Research Institute.

\section{Author contributions}

All authors were involved in conception of experiments or experimental approaches, performing experiments, and writing and proofing the paper.

\section{Additional information}

Supplementary Information accompanies this paper at https://doi.org/10.1038/s41467019-11879-2.

Competing interests: The authors declare no competing interests.

Reprints and permission information is available online at http://npg.nature.com/ reprintsandpermissions/

Peer review information Nature Communications thanks Morgan Huse and Ken Shortman for their contribution to the peer review of this work.

Publisher's note Springer Nature remains neutral with regard to jurisdictional claims in published maps and institutional affiliations.

\begin{abstract}
(c) (i) Open Access This article is licensed under a Creative Commons Attribution 4.0 International License, which permits use, sharing, adaptation, distribution and reproduction in any medium or format, as long as you give appropriate credit to the original author(s) and the source, provide a link to the Creative Commons license, and indicate if changes were made. The images or other third party material in this article are included in the article's Creative Commons license, unless indicated otherwise in a credit line to the material. If material is not included in the article's Creative Commons license and your intended use is not permitted by statutory regulation or exceeds the permitted use, you will need to obtain permission directly from the copyright holder. To view a copy of this license, visit http://creativecommons.org/ licenses/by/4.0/
\end{abstract}

(C) The Author(s) 2019 